\title{
Progressive alterations of striatal polysomal architecture in a mouse model of Huntington's disease
}

Eva Martin-Solana ${ }^{1}$, Irene Diaz-Lopez ${ }^{2}$, Ivan Ventoso ${ }^{3}$, Jose-Jesus Fernandez ${ }^{4,5, *}$, Maria Rosario FernandezFernandez ${ }^{4,5, *}$

${ }^{1}$ Centro Nacional de Biotecnología (CNB-CSIC). Campus UAM, Darwin 3, 28049 Madrid. Spain.

${ }^{2}$ MRC Laboratory of Molecular Biology, Cambridge Biomedical Campus, Cambridge, UK

${ }^{3}$ Centro de Biología Molecular "Severo Ochoa" (CSIC-UAM) and Departamento de Biología Molecular, Universidad Autónoma de Madrid (UAM), Madrid, Spain.

${ }^{4}$ Centro de Investigación en Nanomateriales y Nanotecnología (CINN-CSIC). Av. Vega 4-6. 33940 El Entrego. Asturias, Spain.

${ }^{5}$ Instituto de Investigación Sanitaria del Principado de Asturias (ISPA). Av. Hospital Universitario s/n, 33011 Oviedo, Asturias, Spain.

*Corresponding authors:

JJ.Fernandez@csic.es, MR.Fernandez@csic.es 


\section{Abstract}

Neurons rely on a precise spatial and temporal control of protein synthesis due to their highly polarized morphology and their functional singularities. Consequently, alterations in protein translation have been widely related to the development and progression of various neurological and neurodegenerative disorders, including Huntington's disease. Here we explored the architecture of polysomes in their native brain context by performing 3D electron tomography of striatal tissue derived from a knock-in mouse model of the disease. Results showed a progressive remodelling towards a polysomal compacted architecture that parallels in time the emergence and progression of symptoms in the mouse model. The aberrant architecture is compatible with ribosome stalling phenomena and, in fact, we detected an increase in the expression of the stalling release factor elF5A2. Polysomal sedimentation gradients showed significant excess in the accumulation of free $40 \mathrm{~S}$ ribosomal subunits in heterozygous striatal samples. Overall the results indicate that changes in the architecture of the protein synthesis machinery might be at the basis of translational alterations associated to Huntington's disease and open new avenues for understanding disease progression. 


\section{Introduction}

Huntington's disease (HD) is a genetically inherited dominant neurodegenerative disorder caused by the expansion of a CAG repeat sequence in the coding sequence of huntingtin protein (HTT) (Bates, 2005). Symptoms manifest at mid age (35-45 years old) and life expectancy after the debut of the disease is usually 15 to 20 years. The brain region initially most affected by the disease is the corpus striatum and from a cellular level the neudegeneration selectively affects medium-size spiny neurons (MSSN) that are GABAergic projection neurons (Albin et al, 1992; Vonsattel et al, 1985). Individuals affected by HD develop progressive motor dysfunction, cognitive decline and psychological alteration (Bates et al, 2015; Ross \& Tabrizi, 2011). There is no effective treatment for the disease, although different pharmacological approaches are being explored (Caron et al, 2018; Tabrizi et al, 2019). Recently, promising clinical trials of therapies based on antisense oligonucleotides have been halted in phase III as the potential benefits did not outweight their risks (Kwon, 2021).

The CAG repeat is polymorphic in normal chromosomes and it is expanded in HD-mutated chromosomes ( The Huntington's Disease Collaborative Research Group, 1993). The number of repeats conditions the age of onset and the severity of the disease. The repeat is located in exon1 and codes for a polyglutamine sequence (polyQ) that resides in the $\mathrm{N}$-terminal region of the protein. HTT is a large $348-\mathrm{kDa}$ protein with a considerably degree of conservation from flies to mammals (Saudou \& Humbert, 2016). After the polyQ sequence HTT holds a proline rich region (PRD) that is also polymorphic in the human population (Saudou \& Humbert, 2016). Exon 1 has been poorly conserved during evolution in contrast to other exons. Interestingly the PRD is found only in mammals, suggesting a recent evolution of the HTT protein (Tartari et al, 2008). In yeast systems the presence of the PRD was found to reduce the toxicity of exon1 constructs containing polyQ sequences in the pathological range (Duennwald et al, 2006) and the presence of the PRD has been shown to reduce the rate of polyQ aggregation in vitro (Bhattacharyya et al, 2006; Dehay \& Bertolotti, 2006; Yang et al, 2016; Meriin et al, 2007). Normal 
HTT function and the basis for the toxicity of mutant HTT (mHTT) in the context of HD are still not fully understood (Nowogrodzki, 2018).

As a consequence of their polarized organization and the particularities of synaptic activity, neurons need a precise spatial and temporal control of protein translation. Thus neurons are particularly susceptible to alterations in the control mechanisms of protein translation (Kapur et al, 2017). Deregulation of cellular mechanisms involved in protein synthesis have been widely related to the development and progression of diverse neurological and neurodegenerative diseases (Darnell, 2014; Gao et al, 2017; Kapur \& Ackerman, 2018; Kapur et al, 2017). Precisely, several studies have shown that mutations in proteins involved in sorting out ribosome stalling induce motor dysfunction, neurodegeneration (Chu et al, 2009) and neuronal cell death in mice (Ishimura et al, 2014). There is growing evidence of alterations in protein translation related to $\mathrm{mHTT}$ translation and in the context of HD. Surprisingly, mRNAs containing expanded CAG repeats were proven to be translated more efficiently than those in the non-pathological range (Krauß et al, 2013). The increasing number of CAG repeats favours the binding of a translation regulation complex containing MID1-PP2A. Consequently, the accumulation of the mHTT protein containing polyQ repeats in the pathological range is considerable higher than the non-pathological range ones. These results show an interesting gain of function of the mutant protein at the mRNA level. Different groups have evaluated translation in HD contexts by using the SUnSET method and have obtained sometimes opposite results. The method is based on the ability of puromycin to label the newly synthesized peptides when administrated at low doses. Creus-Muncunill et al. reported an increase in the incorporation of puromycin by striatal cells in brain slices of the R6/1 HD mouse model (CreusMuncunill et al, 2019), pointing to an increase in protein translation and proposing that an overall increase in protein translation could be a novel pathogenic mechanism in HD. Interestingly, the proteomic characterization showed that the translation increase specifically affects a set of proteins related to ribosomal and oxidative phosphorylation while others related to neuronal structure and function are downregulated. On the other side, Joag et al. reported a reduced incorporation of puromycin to Drosophila cells 
overexpressing a fragment of HTT containing a 138 polyglutamine expansion (Joag et al, 2020). The results were also reproduced in SYS5 and Neuro2a cells and suggested that the expression of a pathogenic mHTT fragment causes a protein synthesis deficit. Similarly, a recent report shows that there is less incorporation of puromycin in a heterozygous and homozygous knock-in cellular mouse model STHdh containing 111 CAGs than in the wt counterpart (7 CAGs) supporting the existence of a diminished protein synthesis in HD (Eshraghi et al, 2021). In this context HTT was reported to promote ribosome stalling by binding to ribosomes, an effect that is exacerbated by the mutant version of the protein, mHTT (Eshraghi et al, 2021). In accordance with latter results, the general protein synthesis in yeast cells expressing fragments of pathogenic exon1 was found to be significantly repressed based on the downregulation of expression of genes within the ribosome biogenesis and rRNA processing and metabolism (Tauber et al, 2011). The opposing reports described above may well be due to the different HD models employed or be representative of different disease scenarios. But overall these results show that there is a dysregulation of protein synthesis in the presence of $\mathrm{mHTT}$.

Electron tomography allows the study of 3D subcellular architecture and the organization of molecules in their cellular or tissue native environment with nanometric resolution (Frank, 2006; He \& Fernández, 2010). It is based on the acquisition of images from the specimen at different tilt angles, subsequently processed and combined to yield a 3D reconstruction or tomogram (Fernandez, 2012). Different studies have employed electron tomography to understand the special distribution and interaction that ribosomes established in their cellular native context including bacteria (Brandt et al, 2009), isolated glioblastoma cells (Brandt et al, 2010) or in HeLa cells (Mahamid et al, 2016) and more recently, as a proof of principle, in their tissue context in Caenorhabditis elegans (Schaffer et al, 2019). Rapid-freezing techniques are employed to achieve optimal structural preservation of cells and tissues for electron tomography. Particularly, high-pressure freezing (HPF) is used to fully vitrify bulk specimens (up to 200-400 $\mu \mathrm{m}$ ) by delivering synchronized pressurization and cooling of the sample within milliseconds. Tissue samples can either be processed for observation under cryogenic conditions (Schaffer et al, 2019) or freeze- 
substituted to replace the frozen cellular water with organic solvents and embedded in resins at low temperature to proceed with the analysis at room temperature (He \& Fernández, 2010; Fernandez-Fernandez et al, 2017). Fully cryogenic conditions are ideal to preserve structure in the closest-to-native conditions and to achieve the highest structural resolution, however these protocols are not yet of routine use (Schaffer et al, 2019). Thus, HPF and FS are the combination of choice for a systematic and comparative analysis of tissue ultrastructural alterations in pathological conditions (FernandezFernandez et al, 2017).

Here we explored the architecture of neuronal polysomes in their native brain context by performing 3D electron tomography of HPF/FS striatal tissue derived from the zQ175 knock-in HD mouse model (Menalled et al, 2012). We found a progressive remodelling of polysomal architecture to a densely packed conformation that is compatible with the existence of ribosome stalling phenomena. The increase in the expression of the stalling release factor elF5A2 corroborates this scenario, but the absence of a considerable recruitment of the factor to the polysomal area suggests an inefficient release of the stalling. Polysomal sedimentation gradients also showed remarkable excess in the accumulation of free $40 S$ ribosomal subunits in heterozygous striatal samples. These changes in the architecture of the protein synthesis machinery could be the basis of translational alterations associated to Huntington's disease, besides they open new avenues for understanding disease progression and reinforce protein translation as a potential target for therapeutic intervention.

\section{Materials and Methods}

\section{Animals}

A stable colony of the HD mouse model zQ175 (Menalled et al, 2012) was initiated with founders donated by the Cure Huntington's disease Initiative (CHDI) and obtained from the Jackson Laboratoy Inc. colony. zQ175 is a knockin line on a C57BL/6J background with an endogenous murine HTT gene containing a chimeric human/mouse exon 1 with approximately 190 CAG 
repeats (B6.12951- $\mathrm{Htt}<\mathrm{tm} 1 \mathrm{Mfc}<190 \mathrm{JChdi})$. Mice were bred as a stable colony in heterozygosity in the animal facility of the Centro Nacional de BiotecnologíaCSIC, Madrid with food and water available ad libitum. Experiments complied with Spanish and European legislation and Spanish National Research Council (CSIC) ethics committee on animal experimentation.

\section{Sample preparation based on HPF/FS for electron microscopy}

Brain tissue samples were prepared for electron microscopy (EM) and tomography following our protocols for optimal structural preservation based on high-pressure freezing and freeze-substitution (HPF/FS), as described in (Fernandez-Fernandez et al, 2017). In short, mouse brains were dissected immediately post-mortem and $200 \mu \mathrm{m}$ sagittal slices were obtained by using a tissue slicer (Stoelting, Co.). Striatal samples were promptly extracted, placed on a flat specimen carrier and high-pressure frozen in a Leica EMPACT2 device. The samples were further processed with freeze-substitution of frozen water to methanol and embedded in Lowicryl resin HM20 with a Leica AFS2 EM FSP system. $250 \mathrm{~nm}$ thick sections were obtained from the resin-embedded samples using a Leica Ultracut EM-UC6 ultramicrotome, and placed on Quantifoil S7/2 grids.

\section{Electron microscopy}

A conventional electron microscope JEOL JEM-1011 (100 kV) was used to screen the 250-nm thick sections, check the integrity of the tissue samples and select areas of interest. An average of 5 EM grids, each containing 4 sections, were observed per animal. Animals of 2, 8, 10 and 11 months of age were analyzed. Cells compatible with striatal medium-sized spiny neurons were selected based on morphological criteria (Matamales et al, 2009) (Figure EV1) and representative cytoplasmic areas with abundance of ribosomes were selected for subsequent 3D studies with ET. 


\section{Electron tomography}

Tomographic data were acquired by taking series of images from the sections while tilting them within a range of $\pm 60^{\circ}$ at $1^{\circ}$ interval around a single tilt-axis. The tilt-series were acquired using a Thermo Fisher Scientific Talos Arctica electron microscope (200 kV) equipped with an electron direct detector Falcon II or using a FEI Tecnai G2 (200 kV) equipped with a CCD camera. The pixel size at the specimen level was $0.37 \mathrm{~nm}$ or $0.59 \mathrm{~nm}$, respectively. For processing, visualization and analysis, images were rescaled with a binning factor of 4 . Prior to ET, the grids were incubated in a solution of $10 \mathrm{~nm}$ diameter colloidal gold (EM.BSA 10, Electron Microscopy Sciences, Hatfield, PA, USA) to facilitate subsequent image alignment.

Tilt-series alignment and calculation of the 3D tomograms were conducted with the standard software IMOD (Kremer et al, 1996) using standard protocols (Fernandez, 2012). Images of the tilt-series were mutually aligned using the colloidal gold beads as fiducial markers. Tomograms were reconstructed with the standard method, weighted back-projection (WBP), using a filter simulating iterative methods (SIRT) (Fernandez, 2012).

\section{Computational analysis of the ribosomal pattern in tomograms}

To characterize the ribosomal pattern observed in the tomograms, we developed computational procedures to (1) automatically identify ribosomes and (2) analyze how they are organized. The procedure for identification of ribosomes is based on the Laplacian of Gaussian (LoG), a strategy commonly used in the field of Computer Vision to detect 'blobs' (Nixon and Aguado, 2019). Here, we have extended this strategy to $3 \mathrm{D}$ in order to work with tomograms. Essentially, the procedure applies a Gaussian filtering, with a standard deviation related to the size of the target 3D blob (i.e. the ribosome, around 25 $\mathrm{nm}$ in diameter) and, afterwards, a Laplacian operator (i.e. second-order derivative) is applied to the Gaussian-filtered tomogram. The resulting LoG map shows peaks at the positions where the ribosomes are in the tomogram. The final ribosome positions are extracted by a segmentation operation based on thresholding of the LoG map. 
To analyze the ribosomal organization from the detected ribosome positions, we implemented second-order spatial point pattern analysis techniques extended to 3D. In particular, we used Ripley's K function (Ripley, 1988), K(r), which allows us to measure the expected number of ribosomes within a distance $r$ from an arbitrary ribosome, normalized by the density of the ribosome distribution. This function can be mathematically expressed as:

$$
K(r)=V \cdot \frac{\sum_{i=1}^{n} \sum_{j \neq i} e_{i}(r) \cdot I\left[d_{i j} \leq r\right]}{n^{2}}
$$

where $V$ is the volume of the tomogram, $n$ denotes the number of ribosomes in the tomogram, $d_{i j}$ represents the Euclidean distance from the ribosome $i$ to $j$, I[.] is an indicator function that returns 1 if the distance $d_{i j}$ is within the radius $r$ and 0 otherwise. The term $e_{i}(r)$ is an edge-correction factor to properly weight the contribution of ribosomes with partial neighbourhood due to their proximity to the edge of the tomogram (Ripley, 1988). Ripley's K function is calculated at a range of distances $r$, with a recommended maximum value that should be lower than half of the shortest dimension of the sample domain (Ripley, 1988; Baddeley et al, 1993). In this work, the spatial analysis was applied at distances from $25 \mathrm{~nm}$ (i.e. ribosome diameter) to $100 \mathrm{~nm}$.

Under the hypothesis of complete spatial randomness (CSR), the $\mathrm{K}$ function proves to be equal to the volume of a sphere of radius $r$ (Ripley, 1988; Baddeley et al, 1993), that is, $K_{C S R}(r)=4 / 3 \cdot \pi \cdot r^{3}$. The ratio $K(r) / K_{C S R}(r)$ thus allows analysis of the ribosomal distribution pattern and its classification into the three main categories (Diggle, 2013): aggregation or clustering (if $K(r) / K_{C S R}(r)>$ 1 ), regularity or dispersion (if $K(r) / K_{C S R}(r)<1$ ), or random (if $K(r) / K_{C S R}(r)=1$ ). Supplementary Figure S1 illustrates the calculation of Ripley's K function and the three main spatial distribution patterns.

The analysis of the ribosomal distribution was applied to selected tomograms of 11- month old animals, which were acquired on the FEI Tecnai G2 microscope. The larger field of view of this microscope allowed acquisition of regions with an area up to $2.4 \mu \mathrm{m} \times 2.4 \mu \mathrm{m}$. 


\section{qRT-PCR}

Striatal samples from 12-month-old mice brains were used for gene expression analysis (8 heterozygous zQ175 animals and 8 corresponding matched wt controls). Genes analyzed were: eiF5A1, eiF5A2, DHPS and DOHH and the primers used are described in Supplementary information (table 1). Reference genes were used for normalization of gene expression data (GAPDH, ACTB, $B 2 M, 18 S$ and $Y W H A Z$ (primers in table 2, supplementary information). Suitability of reference genes for normalization of gene expression was evaluated using Normfinder algorithm showing YWHAZ as the most stable and accurate gene for normalization. Total RNA was isolated using Maxwell 16 LEV simply RNA tissue kit (Promega, \#AS1280) and RNA integrity was checked with the Agilent 2100 Bioanalyzer. RIN values were between 7 and 9.9, indicating a very good integrity of the samples. cDNA synthesis was performed using the iScript cDNA Synthesis kit (Bio-rad, \#170 - 8891). qRT-PCRs were performed in triplicate in a CFX384 Real Time System C1000 Thermal Cycler (Bio-rad) using Sso Fast EvaGreen Supermix (Bio-rad, \#172 - 5204). Reactions included non-template control and primer efficiency curves were also performed. ValidPrime kit (TATAA Biocenter, \#A105S10) was used as control for genomic background (gDNA). The contribution of gDNA to qPCR signal was less than $6.5 \%$ in all cases. A melting curve from $60{ }^{\circ} \mathrm{C}$ to $95{ }^{\circ} \mathrm{C}\left(0.5{ }^{\circ} \mathrm{C} / \mathrm{seg}\right)$ was included at the end of the program to verify the specificity of the PCRs. Data processing was carried out using GenEx v5.4.4 (MultiD Analysis AB,

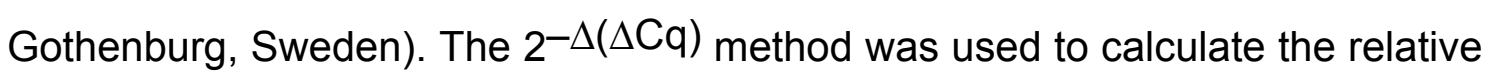
fold gene expression of samples normalized by the YWHAZ gene expression (Livak \& Schmittgen, 2001).

\section{Cell culture and transfection}

HEK293T cells were cultured in DMEM medium (Sigma, \#D6429) supplemented with $10 \%$ inactivated fetal bovine serum, non-essential aminoacids (1:100, Sigma, \#M7145), $0.5 \mu \mathrm{g} / \mathrm{ml}$ Amphotericin B (Fungizone ${ }^{\mathrm{TM}}$ ) (Gibco ${ }^{\mathrm{TM}}$, \#15290018), penicillin-streptomycin (1:100, Sigma, \#P4333) and 50 $\mu \mathrm{g} / \mathrm{ml}$ gentamycin (Sigma, \#G1397). Cells were cultured at $37^{\circ} \mathrm{C}$ in a $5 \% \mathrm{CO}_{2}$ atmosphere. 
pEGFP-Q23 and pEGFP-Q74 plasmid were a gift from David Rubinsztein (Addgene plasmid \# 40261 and 40262) (Narain et al, 1999). They express a fusion protein of GFP to the sequence coded by part of the human HTT exon1. Fusion proteins contain either a non-pathological polyQ repeat $(23 \mathrm{Q})$ or a polyQ repeat in the pathological range $(74 \mathrm{Q})$. These plasmids were co-transfected with $\mathrm{pCMV}-\alpha \mathrm{NP}$ (a plasmid expressing the influenza virus nucleoprotein) as a transfection control (Coloma et al, 2009).

The day before transfection $6 \times 10^{5}$ cells were plated per well in a M6 plate (Falcon, \# 353046) to get to $70-80 \%$ confluence. For actual transfection plasmids were diluted in a solution containing $250 \mathrm{mM} \mathrm{CaCl} 2$ to have a final amount of 1 o $3 \mu \mathrm{g}$ per well (3 $\mathrm{ml}$ final volume per well) for the HTT plasmids and $2 \mu \mathrm{g}$ for the pCMV- $\alpha$ NP. The DNA $\mathrm{CaCl}_{2}$ solution was mixed for 1 min with an equal amount of filtered HBS buffer (50 mM HEPES pH 7.05, $1.5 \mathrm{mM}$ $\mathrm{Na}_{2} \mathrm{HPO}_{4}, 140 \mathrm{mM} \mathrm{NaCl}$ ). Cells were recovered in the culture medium after the corresponding hours of incubation and centrifuged at $1500 \mathrm{rpm}$ for $5 \mathrm{~min}$ at $4^{\circ} \mathrm{C}$. The pellet was then washed with PBS1x and centrifuged as before. The pellet was frozen and kept at $-80^{\circ} \mathrm{C}$. Cell lysis was done by thermal shock ( 3 cycles of $15 \mathrm{~min}$ at $\mathrm{RT}+30 \mathrm{~min}$ at $-80^{\circ} \mathrm{C}$ ) in a buffer containing $50 \mathrm{mM}$ Tris- $\mathrm{HCl} \mathrm{pH} 7.4$ and $150 \mathrm{mM} \mathrm{NaCl}$. The lysate was centrifuged at $16100 \mathrm{~g}$ for $10 \mathrm{~min}$ at $4^{\circ} \mathrm{C}$ and the supernatant use for western-blot analysis as described below. Before the recovery of cells the plates were observed in a Leica DMI6000B microscope at 10x magnification for the detection of GFP-fused proteins.

\section{Polysome sedimentation gradients}

Polysome profiles were obtained as described by (Heiman et al, 2008; Nielsen, 2011; Díaz-López et al, 2019) with some modifications. Briefly, the mouse brain regions of interest (striatum or hippocampus) were dissected and immediately frozen in liquid nitrogen. Samples from matched genotype animals were pooled to overcome the limitation of the low sample weight (for each genotype four animals). Frozen samples were ground with a mortar and pestle under liquid nitrogen and homogenized in ice-cold polysome extraction buffer $(20 \mathrm{mM}$ HEPES pH 7.4, 150 mM KCl, 5 mM MgCl $2,0.5$ mM DTT, 1\% Triton X-100, 200 $\mu \mathrm{g} / \mathrm{ml}$ cycloheximide $(\mathrm{CHX})$, heparin $20 \mu \mathrm{g} / \mathrm{ml}$ and $40 \mathrm{U} / \mathrm{ml}$ RNase inhibitor 
(New England Biolabs, Cat. no. M0307S) using a potter homogenizer (10-12 strokes). After three passages through a $23 G$ needle, the homogenates were centrifuged at $2000 \mathrm{~g}$ for $5 \mathrm{~min}$ at $4^{\circ} \mathrm{C}$. The supernatants were subsequently centrifuged twice at $16100 \mathrm{~g}$ for $10 \mathrm{~min}$ at $4^{\circ} \mathrm{C}$. The final supernatants $(600 \mu \mathrm{l})$ were loaded onto $10-50 \%$ continuous sucrose density gradients (20 mM HEPES [pH 7.4], 150 mM KCl, 5 mM MgCl 2 ) (Gradient Master ${ }^{\text {TM }}$, BioComp) and centrifuged in a SW40Ti swing-out rotor (Beckman Coulter) at 37500 rpm for 2 $\mathrm{h}$ at $4^{\circ} \mathrm{C}$. One $\mathrm{ml}$ fractions were collected using a density gradient fractionation system (ISCO) with continuous monitoring of absorbance at $254 \mathrm{~nm}$.

Polysomal sedimentation gradients of transfected cells were performed as described by (Pringle et al, 2019), with some modifications. Briefly, $24 \mathrm{~h}$ post transfection, HEK293T cells were treated with $100 \mu \mathrm{g} / \mathrm{ml} \mathrm{CHX}$ for $3 \mathrm{~min}$ at $37^{\circ} \mathrm{C}$. Cells were then washed 3 times with cold $\mathrm{CHX}(100 \mu \mathrm{g} / \mathrm{ml})$ in PBS (1x), harvested with residual $\mathrm{CHX} / \mathrm{PBS}$ and centrifuged at $1000 \mathrm{~g}$ for $5 \mathrm{~min}$ at $4^{\circ} \mathrm{C}$. The cell pellet was washed with ice-cold CHX/PBS, centrifuged at $1000 \mathrm{~g}$ for 5 min at $4^{\circ} \mathrm{C}$ and resuspended in $500 \mu$ of polysome extraction buffer $(100 \mu \mathrm{g} / \mathrm{ml}$ $\mathrm{CHX}$ and no heparin). The cell lysate was mixed through a $23 \mathrm{G}$ needle and incubated on ice for $10 \mathrm{~min}$ (vortexing every 2-3 min). The homogenates were centrifuged at $2000 \mathrm{~g}$ for $5 \mathrm{~min}$ at $4^{\circ} \mathrm{C}$ and subsequently centrifuged twice at $16100 \mathrm{~g}$ for $10 \mathrm{~min}$ at $4{ }^{\circ} \mathrm{C}$. The final supernatants $400 \mu \mathrm{l}$ were loaded onto a $10-50 \%$ continuous sucrose density gradient (Gradient Station ${ }^{\mathrm{TM}}$, BioComp) and centrifuged in an SW40Ti swing-out rotor at $37500 \mathrm{rpm}$ for $2 \mathrm{~h}$ at $4^{\circ} \mathrm{C}$. One $\mathrm{ml}$ fractions were collected manually.

\section{Western-blot analysis.}

Bulk striatal samples were processed for western-blot as described previously (Fernandez-Fernandez et al, 2011), seven 12-month old heterozygous animals and seven corresponding wt controls were used. Samples were loaded onto NuPAGE 12\% Bis-Tris gels (Invitrogen, NP0343) and run in MES running buffer (Invitrogen, NP0002). Proteins were transferred to nitrocellulose membranes (BioRad, 1620112). Membranes were incubated with the following antibodies 
anti-hypusine (ABS1064, Merck), anti-elF5A1 (SAB1402762, Sigma-Aldrich, Merck) and anti-elF5A2 (GTX116635, Genetex).

Fractions from sucrose gradients were loaded and transfer as described above. Membranes were incubated with the following primary antibodies: anti-RPS6 (Elabscience, E-AB-32813), anti-RPL7 (Elabscience, E-AB-32805) and antihypusine (Creative Biolabs, PABL-202). For accurate quantitative comparisons membranes were first incubated with the anti-RPS6 antibody and after completing the western-blot membranes were consecutively incubated with anti-RPL7 antibody, for this reason the imaging of the RPL7 signal stills shows the residual signal of anti-RPS6 antibody.

Samples from HEK293T transfected cells were processed as shown above and membranes were incubated consecutively with anti-GFP (Genetex \#GTX113617) and anti- $\alpha$ NP (Coloma et al, 2009).

The secondary antibodies used were AffiniPure Goat anti-rabbit $\lg G(\mathrm{H}+\mathrm{L})$ (Jackson ImmunoResearch, 111-035-003) and AffiniPure Goat anti-mouse IgG $(\mathrm{H}+\mathrm{L})$ (Jackson ImmunoResearch, 115-035-003). All antibodies were used at 1:1000 dilutions. The peroxidase reaction was developed with Clarity ${ }^{\mathrm{TM}}$ western ECL substrate (Bio-Rad, 1705061). The imaging was performed either in an Odyssey Fc imaging system (Li-Cor) (quantified using Image StudioTM software) or in a Molecular ChemiDoc ${ }^{\mathrm{TM}}$ Touch Imaging System (Bio-Rad) (quantified with the Image Lab 6.0.1 software).

\section{Statistical analysis}

Statistical analyses were performed using GrapPad Prism 8. The normal distribution of the data was checked by means of Shapiro-Wilk test. Data were compared with two-tailed unpaired student t-test when they followed a normal distribution (after testing the equality of variances with Levene test and/or using the Welch's correction). When the distribution of data was not normal, the values were compared with the Mann-Whitney test for non-parametric samples. The level of significance was set at $p<0.05$. All data are represented as mean \pm 
SD with the exception of Figure 5D where the SEM is represented, these latter data were not statistically analysed as $n=2$.

\section{Results}

\section{Ribosomal/polysomal organization is altered in striatal medium-sized spiny neurons}

HD involves the selective degeneration of the striatal MSSN. We aimed to study the architecture of the protein synthesis machinery in their native cellular context with electron tomography to identify and characterize alterations in these neurons.

Brain tissue samples from HD animal models (heterozygous zQ175) at different ages (2, 8, 10, 11 months old) and the corresponding controls were prepared with HPF/FS for electron microscopy and tomography, as described in Materials and Methods. This preparation protocol ensures optimal structural preservation of tissue samples. Conventional bidimensional (2D) electron microscopy of 250$\mathrm{nm}$ thick sections was used to confirm the integrity of the samples and to select areas of interest. We selected representative cytoplasmic areas from the soma of cells compatible with being striatal MSSN where ribosomal and polysomal crowding was identified, and they were subjected to electron tomography to compute 3D volumes or tomograms.

Figures EV2 and EV3 show representative EM images and slices of 3D volumes obtained from a wt control and a HD model at advanced age. In wt samples the ribosomes are abundant, show a continuous distribution across the entire cytoplasm and form polysomal clusters where individual ribosomes can be clearly identified. By contrast, in HD model the ribosomal density is reduced and the polysomes appear as forming sparse clusters of tightly-coupled ribosomes with large empty cytoplasmic space between the clusters. Figure 1 shows a gallery of representative tomographic slices from animals at different ages where this distinctive pattern in HD is observed to increase with age. Altogether, these results indicate that in HD the remodelling of polysomal 
architecture starts to be evident at ages (8 months) when some of the relevant phenotypes of the model (decrease in rotarod performance or striatal atrophy) (Menalled et al, 2012) debut and most interestingly the alterations in polysomal architecture show a clear progression with age.

In order to conduct an objective quantitative analysis, we developed a methodology for automated identification of ribosomes in the tomograms followed by a second-order spatial analysis using Ripley's K function, as described in Materials and Methods. Figures EV2D and EV3D illustrate the detection of ribosomes in the respective tomograms of the wt control and the HD model. Those panels further show that the cytoplasm of the wt animals is densely populated with ribosomes whereas in the HD they are rather organized in groups.

The spatial analysis was applied to the ribosome positions detected in selected tomograms (5 wt and 6 heterozygous) of different neurons from 11-month old animals. Ripley's K-function $K(r)$ estimates the number of ribosomes around an arbitrary one within a distance $r$, normalized by the ribosomal density. The plots in Figure 2 represent Ripley's K-function relative to a random distribution $\left(K(r) / K_{C S R}(r)\right.$, see Materials and Methods). Thus, a unit value indicates a random distribution whereas values higher than 1 suggest clustering. The functions show that the ribosomes are significantly more clustered in the heterozygous HD model $\left(K(r) / K_{C S R}(r)\right.$ much higher than 1$)$ than in the wt control, especially at short distance (up to $50 \mathrm{~nm}$ ). The wt phenotype also shows some ribosome clustering, though much weaker than in the HD model, and this is consistent with the fact that ribosomes form polysomes for the sake of efficient translation. We also calculated the ribosomal density (detected ribosomes per volume unit) and it turned out to be higher in the wt control than in the HD model (4955 vs. 2623 ribosomes $/ \mu \mathrm{m}^{3}$ ). Therefore, this objective analysis reflects in quantitative terms the lower ribosomal density and the compact clustering of the ribosomes observed in the tomograms, thereby confirming the ribosomal alterations in the HD model. 


\section{The elF5A2 stalling release factor is overexpressed in the striatum of heterozygous zQ175 animals}

The phenotype observed in polysomal architecture in the HD model is reminiscent of ribosome stalling phenomena. It is well established that the presence of consecutive prolines in a polypeptide, as it is the case of the PRD region in HTT, induces ribosome stalling. Proline is neither a good donor nor a good acceptor during the formation of the peptide bond, as the reactive amine is embedded in the cyclic lateral chain, thus reducing its nucleophilicity (Wohlgemuth et al, 2008; Pavlov et al, 2009; Melnikov et al, 2016). elF5A, and its homologue in bacteria EF-P, has been identified as a factor involved in stimulating the formation of the peptide bond between consecutive prolines, and thus releasing the ribosome stalling (Doerfel et al, 2013; Gutierrez et al, 2013; Ude et al, 2013).

elF5A is coded by two different genes giving rise to two different homologous proteins, elF5A1 and elF5A2 (Wu et al, 2020). While elF5A1 is widely expressed in all tissues, the expression of elF5A2 is weak in the majority of tissues with the exception of the brain and testis where levels are high (Clement et al, 2006). elF5A proteins are the only known proteins containing the aminoacid hypusine, which is a posttranslational modification of lysine. Hypusine is essential for elF5A function in releasing ribosome stalling (Melnikov et al, 2016; Saini et al, 2009; Schmidt et al, 2016). The formation of hypusine involves two enzymatic reactions (Park et al, 1981; Schmidt et al, 2016), first deoxyhypusine synthase (DHS) produces deoxyhypusine, which is then hydroxylated by deoxyhypusine hydroxylase $(\mathrm{DOHH})$ to produce hypusine. We explored a potential activation of the elF5A pathway that might be specifically induced in the heterozygous models by the PRD sequence in HTT. We analysed the expression of elF5A factors and related genes in the striatum of heterozygous 12-month old animals and their corresponding wt controls by qRT-PCR. Figure $3 A$ shows that there is no significant difference in the expression of genes coding for elF5A1, DHS or $\mathrm{DOHH}$, while there is a significant increase in the expression of elF5A2 in the heterozygous animals. The overexpression of the brain enriched elF5A2 factor could be reflective of a 
response to release stalling in HD conditions. It is important to note that the analysis in bulk of the striatum could be occulting or underestimating differences that might be specific to MSSN.

We then explored the accumulation of elF5A1 and elF5A2 proteins and their hypusinated forms by western-blot. We have not been able to detect any significant difference in the accumulation of any of them in the striatum of heterozygous zQ175 animals as compared to their corresponding wt controls at the age of 12 months (Figure 3B). elF5A1 and elF5A2 are 84\% identical (Clement et al, 2006) with the stretch spanning from aa 19 to 88 being fully identical, they have a molecular weight of $17 \mathrm{kDa}$. We can only detect a single band at that size with an anti-hypusine antibody (Figure $3 B$ ). While some authors have been able to electrophoretically separate elF5A1 and elF5A2, we have not been able to find any condition where we could separate them (Figure 3B). Thus, the presence of a single band in the incubation with the antihypusine antibody could contain a mixture of both hypusinated forms impeding the quantification of particular differences in the two isoforms. The high degree of identity of both proteins and the insufficient information of the epitope recognized by the different commercial antibodies precludes a conclusive interpretation about the bands detected by the anti-elF5A1 and anti-elF5A2 antibodies, and a cross-reaction cannot be initially discarded. Thus these limitations in the western-blot analysis make difficult to extrapolate the effect that the differences observed in the expression of elF5A2 may have at the protein level and in the degree of hypusination.

Despite being unable to detect any significant difference in the accumulation of the elF5A stalling release factors, we decided to explore if there was any differential engagement of the active (hypusinated) form to ribosomes. We optimized a protocol for preparing polysomal sedimentation gradients, given the small weight of the mouse striatum the protocol had to be performed with a pool of samples from 4 animals. Figure $4 \mathrm{~A}$ shows the profiles obtained for the wt and heterozygous zQ175 pools. The peaks corresponding to the $40 S$ (small subunit), 605 (large subunit), 805 (monosome) and polysomes are clearly identifiable in the profiles. The analysis of the fractions by western-blot (Figure 
4B) confirmed the expected distribution of ribosomal proteins corresponding to the small and large subunits (RPS6 and RPL7), respectively. Surprisingly, in both genotypes, most of the hypusinated elF5A factor $(>80 \%)$ is found in fractions 1 and 2 that do not contain ribosomal subunits. Thus most of the active stalling release factor is found not associated to ribosomes. This result can be consistent with a transient interaction of the factor with the 605 subunit (Rossi et al, 2016) or be indicative of alternative functions of the elF5A factor other than facilitating elongation in polyproline induced stalling, as has been proposed before (Saini et al, 2009; Schuller \& Green, 2018; Schuller et al, 2017; Pelechano \& Alepuz, 2017). Although the distribution of the hypusinated elF5A factor shows slight differences in the first fractions of wt and hetero gradients, it is clear that there is not an increased recruitment of the activated factor to the second half of the heterozygous gradient, where the stalled polysomes would be expected to sediment (from fraction 7 onwards).

\section{There is an excess accumulation of free $40 \mathrm{~S}$ subunit in the striatum of heterozygous zQ175 animals}

The overall observation of profiles from polysomal sedimentation gradients (Figure 4B) showed a very similar overall profile between wt and heterozygous samples, with initially no apparent difference in the polysomal area that could be indicative of the compactation phenotype observed by ET. Despite succeeding in the obtaining of these profiles, the nature of the brain samples interferes with getting optimal clean and concentrated input samples. For this reason, the 40S, 60S and $80 \mathrm{~S}$ peaks are not fully resolved and that first part of the gradient has a considerable background. As the background does not necessarily have to be the same for the wt and the heterozygous sample, and from experiment to experiment, this precludes a quantitative analysis of the profiles to explore difference in the ratios of the different peaks. Despite this limitation the observation of the profiles suggests that the $40 S / 80 S$ ratio is higher in the heterozygous profile. To confirm this we analysed the fractions from the wt and heterozygous profiles by WB using anti-RPS6 protein (small subunit) and anti-RPL7 protein (large subunit) antibodies. In these gradients, RPS6 starts being detected in fraction 3 while RPL7 starts to be detected in 
fraction 4 . This confirms that fraction 3 contains exclusively $40 \mathrm{~S}$ subunits. The observation of western-blots confirmed that the proportion of free 40S (RPS6 in fraction 3) seems higher in the heterozygous gradient in relation to adjacent fractions that in sum contain $60 S$ and 80 subunits (fraction 4 and fraction 5 ). Interestingly, the distribution of ribosomal proteins seems different in the polysomal area, while in the heterozygous samples there is a maximum peak in fraction 11 (both with RPS6 and RPL7) in the case of the wt it looks like the proteins are more widely distributed among surrounding fractions with fraction 10 having equivalent amounts to fraction 11. As fractions from these gradients do not share a quantitative control for normalization, we divided the gradients in two halves and loaded wt and heterozygous samples in the same gel (fraction 1 to 7 and fraction 7 to 13). Figure $4 \mathrm{C}$ shows a representative WB of the first half of the gradient. For a comparative analysis of wt and heterozygous distribution of proteins, we calculated the percentage of accumulation of each ribosomal protein present in a particular fraction $(100 \%$ is the sum of the intensity for a given protein RPS6 or RPL7 in each genotype). We did this analysis in triplicate (three independent western-blot to compensate for error in pipetting). The graphs show the average percentage of accumulation $\pm \mathrm{SD}$. While the percentages of accumulation of RPL7 protein in both wt and heterozygous samples are highly overlapping, the RPS6 percentages vary considerably between wt and heterozygous fractions. The increase in the percentage of accumulation of RPS6 in fraction 3 is significantly higher in heterozygous animals while it is significantly lower in fraction 5 . This result confirms that in heterozygous samples there is an increase ratio of free 40S subunit. Figure 4D shows an equivalent analysis for fractions 7 to 13. For each genotype the percentages of accumulation of RPS6 and RPL7 proteins are very similar, as expected for fractions that contain full ribosomes in different polysomal combinations. However, and to confirm the observation in Figure 4B, the distribution of ribosomal proteins differs between the wt and the heterozygous, with the heterozygous fractions having a clear maximum peak of accumulation in fraction 11. The relevance of this differential distribution of polysomes and the relation that it can have to the architectural phenotype detected by ET are still to be determined. 


\section{The expanded CAG repeat does not have a negative effect on protein accumulation}

Next we wanted to explore if differences in the ribosomal and polysomal organization found in the striatum of animal models are a direct consequence of the mHTT expression. We transfected HEK293 cells with plasmids to overexpress GFP protein fused to the sequence encoded by part of HTT exon1 containing either 23 CAG (pEGFP-Q23) or 74 CAG repeats (pEGFP-Q74), being respectively representative of non-pathological and pathological conditions (Narain et al, 1999). Different concentrations of the plasmids were used ( $1 \mu \mathrm{g}$ and $3 \mu \mathrm{g}$ per well). As a control of transfection, cells were cotransfected with the paNP plasmid (Coloma et al, 2009). Results in Figure 5A and 5B show that higher amounts of plasmid derive in higher accumulation of the fusion proteins for both plasmids. This difference can be due to an increase in the percentage of transfection (3.5\% for $1 \mu \mathrm{g}$ and $5 \%$ for $3 \mu \mathrm{g}$ ) (Figure $5 \mathrm{C}$ ). Interestingly, at both plasmid concentrations the accumulation of the GFP-Q74 fusion protein is higher than the accumulation of GFP-Q23 (statistically significant at $3 \mu \mathrm{g}$ condition). A similar effect had previously been observed by (Krauß et al, 2013) in transfection experiments using mouse embryonic fibroblasts and plasmids that express $17 \mathrm{Q}$ and $49 \mathrm{Q}$ tracks in the context of a wider N-terminal HTT fragment (first 500 aminoacids). They showed that there was no difference in the expression of the mRNAs coding for the Q17 and Q49, reinforcing that differences in the translation efficiency underlie the accumulation effect. They also showed that the increasing number of CAG repeats favours the binding of the MID1-PP2A-containing translation regulation complex. Our results confirm that the effect is highly dependent on the CAG sequence coding for the polyQ as the plasmids we use contain the CAG repeat flanked by a partial sequence coded by exon 1 (10 aa flanking aa on the $\mathrm{N}$ terminus and 17 on the C-terminus).

The overexpression of constructs containing CAG repeats in the pathological range is sufficient to induce the increase in the accumulation of free 405 ribosomal subunits 
To explore if the differences in the polysome sedimentation gradients observed in striatal samples of heterozygous animals are a direct effect of the expression of mHTT, we performed polysomal sedimentation gradients from cells transfected with pEFGFP-Q23 and pEGFP-Q74 at 24 hours post-transfection. Gradients were performed with the slight modifications described in Materials and Methods, meaning that fractions are not fully equivalent to the ones in striatal gradients. Figure 5D shows that the gradients from cells overexpressing GFP-Q74 (pathogenic range) show peculiarities similar to the ones observed in the gradients from heterozygous striatal samples. Mainly involving a displacement of RPS6 to fractions where $40 S$ is expected to be present based on the accumulation of ribosomal proteins. Comparison of the percentages of RPS6 accumulation confirms that the overexpression of GFP-Q74 induces an increased accumulation of free $40 S$ subunit. Additionally, in the intermediate polysomal area fractions the percentage of accumulation of ribosomal proteins is different between cells overexpressing both versions of the fusion protein. While in GFP-Q74 overexpressing cells there is a maximum peak of accumulation around fraction 11, in GFPQ23 overexpressing ones the distribution of ribosomal proteins is more widely distributed, resembling the profiles of accumulation detected in wt and heterozygous striatal samples.

\section{DISCUSSION}

One of the most intriguing characteristics of HD is the fact of its slowly progressive nature. While the mutant protein is expressed throughout life, symptoms manifest at mid age (35-45 years of age) and afterwards life expectancy is around 15-20 years. It has thus been proposed that subtle alterations at the cellular level are likely to accumulate overtime to become detrimental at later stages (Saudou \& Humbert, 2016). Our electron tomography results show a progressive disturbance of neuronal polysomal architecture in the striatum of the zQ175 HD model. In this model, motor coordination phenotypes, measured by rotarod performance, and striatal atrophy start to be detected at 8 months of age (Menalled et al, 2012). Interestingly, it is at 8 months the youngest age in which rearrangements of the polysome structure are evident. This coincidence in time, and the importance of protein synthesis 
for cellular homeostasis, suggest that the polysomal alterations described here might have relevance in the development of HD-related phenotypes in the mice and be a reflection of disease progression. The evidences of disturbances in protein synthesis associated to HD (Creus-Muncunill et al, 2019; Joag et al, 2020; Eshraghi et al, 2021) have driven to propose that translational dysfunction is a contributor to the pathogenesis of HD and to suggest that new therapies targeting protein synthesis might contribute to alleviate disease symptoms (Joag et al, 2020). Our results contribute to reinforce the role of translational dysfunction in HD and point to alterations in the architecture of the protein synthesis machinery as the starting mechanism for translational dysfunction.

Studies based on gene-specific deletion mutants allowed the idenfitication of yeast strains where the toxicity of mHTT is suppressed (Tauber et al, 2011). Interestingtly, all these strains show an increased expression of the stalling release factor elF5A2 when $\mathrm{mHTT}$ is overexpressed. Thereby, the increased expression of elF5A2 we detected in the striatum of $z Q 175$ heterozygous animals could represent a cellular response to the toxicity of mHTT. The increase in elF5A2 expression together with the progressive compactation of polysomes support an scenario of ribosome stalling induced by $\mathrm{mHTT}$. We do not detect an increase recruitment of the hypusinated elF5A factor to polysomal fractions in heterozygous samples, which might imply that a lack of efficient engagement of elF5A to polysomes might prevent an efficient release of the stalling. However, it is important to note that both qRT-PCR experiments and polysome sedimentation gradients have been performed with the whole striatum and, consequently, differences specific to MSSN, the neurons most affected in HD, might be hindered or underestimated by the bulk analysis.

elF5A factors are mainly involved in releasing the ribosome stalling induced by the translation of consecutive prolines (Doerfel et al, 2013; Gutierrez et al, 2013; Ude et al, 2013). Interestingly, the polyQ sequence in HTT is followed by a PRD abundant in consecutive prolines. This PRD is present in both the wt and the mutant version of HTT, opening the question of why the stalling induced by the translation of the PRD should only become evident in heterozygous animals. An interesting study showed that the dependence on the elF5A factors 
for stalling release correlates with the rate of translation initiation of each particular mRNA (Hersch et al, 2014). Thus, when initiation rates are low, translation through polyproline sequences resumes without impeding the progression of upstream ribosomes, even in the absence of the elF5A factors. However, in transcripts that have a high initiation rate, transcripts will often engage simultaneously with several ribosomes that are likely to stall when translating the polyproline sequence and become dependent on the action of elF5A factors. This is very relevant in the context of the results obtained by Krauß et al. demonstrating that the increasing number of CAG repeats favours the binding of the MID1-PP2A-containing translation regulation complex and thus are more efficiently translated than those in the non-pathological range (Krauß et al, 2013). All these evidences suggest that mHTT mRNA as a consequence of being more efficiently translated could be more sensitive to the effect of polyproline derived stalling and thus more dependent on the action of the elF5A release factors.

We have detected an excess accumulation of free $40 S$ subunits in the striatum of the heterozygous zQ175 mouse model. The stability and accumulation of small and large ribosomal subunits are coupled as part of a process that might have evolved to secure proper translational performance. An excess of $40 \mathrm{~S}$ subunits could lead to the formation of translation initiation complexes that cannot be converted to fully translating $80 \mathrm{~S}$ ribosomes and thus sequester mRNAs and induce a general distortion of protein synthesis (Gregory et al, 2019). Thus it would be interesting to explore the consequences of this excess 40S accumulation for striatal dysfunction in HD and how it relates to a potential inefficient release of ribosome stalling induced by $\mathrm{mHTT}$. 


\section{Bibliography}

Albin RL, Reiner A, Anderson KD, Dure LS, Handelin B, Balfour R, Whetsell WO, Penney JB \& Young AB (1992) Preferential loss of striato-external pallidal projection neurons in presymptomatic Huntington's disease. Ann Neurol 31: 425-430

Baddeley AJ, Moyeed RA, Howard CV \& Boyde A (1993) Analysis of a ThreeDimensional Point Pattern with Replication. Appl Stat 42: 641

Bates GP (2005) History of genetic disease: the molecular genetics of Huntington disease - a history. Nat Rev Genet 6: 766-773

Bates GP, Dorsey R, Gusella JF, Hayden MR, Kay C, Leavitt BR, Nance M, Ross CA, Scahill RI, Wetzel R, et al (2015) Huntington disease. Nat Rev Dis Primer 1: 15005

Bhattacharyya A, Thakur AK, Chellgren VM, Thiagarajan G, Williams AD, Chellgren BW, Creamer TP \& Wetzel R (2006) Oligoproline effects on polyglutamine conformation and aggregation. J Mol Biol 355: 524-535

Brandt F, Carlson L-A, Hartl FU, Baumeister W \& Grünewald K (2010) The Three-Dimensional Organization of Polyribosomes in Intact Human Cells. Mol Cell 39: 560-569

Brandt F, Etchells SA, Ortiz JO, Elcock AH, Hartl FU \& Baumeister W (2009) The Native 3D Organization of Bacterial Polysomes. Cell 136: 261-271

Caron NS, Dorsey ER \& Hayden MR (2018) Therapeutic approaches to Huntington disease: from the bench to the clinic. Nat Rev Drug Discov 17: $729-750$

Chu J, Hong NA, Masuda CA, Jenkins BV, Nelms KA, Goodnow CC, Glynne RJ, Wu H, Masliah E, Joazeiro CAP, et al (2009) A mouse forward genetics screen identifies LISTERIN as an E3 ubiquitin ligase involved in neurodegeneration. Proc Natl Acad Sci 106: 2097-2103

Clement PMJ, Johansson HE, Wolff EC \& Park MH (2006) Differential expression of elF5A-1 and elF5A-2 in human cancer cells. FEBS $J$ 273: 1102-1114

Coloma R, Valpuesta JM, Arranz R, Carrascosa JL, Ortín J \& Martín-Benito J (2009) The Structure of a Biologically Active Influenza Virus Ribonucleoprotein Complex. PLoS Pathog 5: e1000491 
Creus-Muncunill J, Badillos-Rodríguez R, Garcia-Forn M, Masana M, GarciaDíaz Barriga G, Guisado-Corcoll A, Alberch J, Malagelada C, DelgadoGarcía JM, Gruart A, et al (2019) Increased translation as a novel pathogenic mechanism in Huntington's disease. Brain 142: 3158-3175

Darnell JC (2014) Ribosome rescue and neurodegeneration. Science 345: 378379

Dehay B \& Bertolotti A (2006) Critical role of the proline-rich region in Huntingtin for aggregation and cytotoxicity in yeast. J Biol Chem 281: 35608-35615

Díaz-López I, Toribio R, Berlanga JJ \& Ventoso I (2019) An mRNA-binding channel in the ES6S region of the translation 48S-PIC promotes RNA unwinding and scanning. eLife 8: e48246

Diggle PJ (2013) Statistical Analysis of Spatial and Spatio-Temporal Point Patterns 0 ed. Chapman and Hall/CRC

Doerfel LK, Wohlgemuth I, Kothe C, Peske F, Urlaub H \& Rodnina MV (2013) EF-P Is Essential for Rapid Synthesis of Proteins Containing Consecutive Proline Residues. Science 339: 85-88

Duennwald ML, Jagadish S, Muchowski PJ \& Lindquist S (2006) Flanking sequences profoundly alter polyglutamine toxicity in yeast. Proc Natl Acad Sci U S A 103: 11045-11050

Eshraghi M, Karunadharma PP, Blin J, Shahani N, Ricci EP, Michel A, Urban NT, Galli N, Sharma M, Ramírez-Jarquín UN, et al (2021) Mutant Huntingtin stalls ribosomes and represses protein synthesis in a cellular model of Huntington disease. Nat Commun 12: 1461

Fernandez J-J (2012) Computational methods for electron tomography. Micron 43: 1010-1030

Fernandez-Fernandez MR, Ferrer I \& Lucas JJ (2011) Impaired ATF6a processing, decreased Rheb and neuronal cell cycle re-entry in Huntington's disease. Neurobiol Dis 41: 23-32

Fernandez-Fernandez MR, Ruiz-Garcia D, Martin-Solana E, Chichon FJ, Carrascosa JL \& Fernandez J-J (2016) 3D electron tomography of brain tissue unveils distinct Golgi structures that sequester cytoplasmic contents in neurons. J Cell Sci: jcs.188060

Frank J ed. (2006) Electron Tomography New York, NY: Springer New York 
Gao F-B, Richter JD \& Cleveland DW (2017) Rethinking Unconventional Translation in Neurodegeneration. Cell 171: 994-1000

Gregory B, Rahman N, Bommakanti A, Shamsuzzaman M, Thapa M, Lescure A, Zengel JM \& Lindahl L (2019) The small and large ribosomal subunits depend on each other for stability and accumulation. Life Sci Alliance 2: e201800150

Gutierrez E, Shin B-S, Woolstenhulme CJ, Kim J-R, Saini P, Buskirk AR \& Dever TE (2013) elF5A Promotes Translation of Polyproline Motifs. Mol Cell 51: 35-45

He W \& Fernández J-J (2010) Electron Tomography. In Encyclopedia of Life Sciences, John Wiley \& Sons, Ltd (ed) p a0021877. Chichester, UK: John Wiley \& Sons, Ltd

Heiman M, Schaefer A, Gong S, Peterson JD, Day M, Ramsey KE, SuárezFariñas M, Schwarz C, Stephan DA, Surmeier DJ, et al (2008) A Translational Profiling Approach for the Molecular Characterization of CNS Cell Types. Cell 135: 738-748

Hersch SJ, Elgamal S, Katz A, Ibba M \& Navarre WW (2014) Translation Initiation Rate Determines the Impact of Ribosome Stalling on Bacterial Protein Synthesis. J Biol Chem 289: 28160-28171

Ishimura R, Nagy G, Dotu I, Zhou H, Yang X-L, Schimmel P, Senju S, Nishimura Y, Chuang JH \& Ackerman SL (2014) Ribosome stalling induced by mutation of a CNS-specific tRNA causes neurodegeneration. Science 345: 455-459

Joag H, Ghatpande V, Desai M, Sarkar M, Raina A, Shinde M, Chitale R, Deo A, Bose T \& Majumdar A (2020) A role of cellular translation regulation associated with toxic Huntingtin protein. Cell Mol Life Sci 77: 3657-3670

Kapur M \& Ackerman SL (2018) mRNA Translation Gone Awry: Translation Fidelity and Neurological Disease. Trends Genet 34: 218-231

Kapur M, Monaghan CE \& Ackerman SL (2017) Regulation of mRNA Translation in Neurons-A Matter of Life and Death. Neuron 96: 616637

Krauß S, Griesche N, Jastrzebska E, Chen C, Rutschow D, Achmüller C, Dorn S, Boesch SM, Lalowski M, Wanker E, et al (2013) Translation of HTT mRNA with expanded CAG repeats is regulated by the MID1-PP2A protein complex. Nat Commun 4: 1511 
Kremer JR, Mastronarde DN \& Mclntosh JR (1996) Computer Visualization of Three-Dimensional Image Data Using IMOD. J Struct Biol 116: 71-76

Kwon D (2021) Failure of genetic therapies for Huntington's devastates community. Nature 593: 180-180

Livak KJ \& Schmittgen TD (2001) Analysis of Relative Gene Expression Data Using Real-Time Quantitative PCR and the 2- $\Delta \Delta \mathrm{CT}$ Method. Methods 25: $402-408$

Mahamid J, Pfeffer S, Schaffer M, Villa E, Danev R, Kuhn Cuellar L, Forster F, Hyman AA, Plitzko JM \& Baumeister W (2016) Visualizing the molecular sociology at the HeLa cell nuclear periphery. Science 351: 969-972

Matamales M, Bertran-Gonzalez J, Salomon L, Degos B, Deniau J-M, Valjent E, Hervé D \& Girault J-A (2009) Striatal Medium-Sized Spiny Neurons: Identification by Nuclear Staining and Study of Neuronal Subpopulations in BAC Transgenic Mice. PLoS ONE 4: e4770

Melnikov S, Mailliot J, Rigger L, Neuner S, Shin B, Yusupova G, Dever TE, Micura R \& Yusupov M (2016) Molecular insights into protein synthesis with proline residues. EMBO Rep 17: 1776-1784

Menalled LB, Kudwa AE, Miller S, Fitzpatrick J, Watson-Johnson J, Keating N, Ruiz M, Mushlin R, Alosio W, McConnell K, et al (2012) Comprehensive behavioral and molecular characterization of a new knock-in mouse model of Huntington's disease: zQ175. PloS One 7: e49838

Meriin AB, Zhang $X$, Alexandrov IM, Salnikova AB, Ter-Avanesian MD, Chernoff YO \& Sherman MY (2007) Endocytosis machinery is involved in aggregation of proteins with expanded polyglutamine domains. FASEB $J$ Off Publ Fed Am Soc Exp Biol 21: 1915-1925

Narain Y, Wyttenbach A, Rankin J, Furlong RA \& Rubinsztein DC (1999) A molecular investigation of true dominance in Huntington's disease. J Med Genet 36: 739-746

Nielsen H ed. (2011) RNA: methods and protocols New York: Humana Press

Nixon M \& Aguado A (2019) Feature extraction and image processing for computer vision. Academic Press.

Nowogrodzki A (2018) Huntington's disease: 4 big questions. Nature 557: S48 
Park MH, Cooper HL \& Folk JE (1981) Identification of hypusine, an unusual amino acid, in a protein from human lymphocytes and of spermidine as its biosynthetic precursor. Proc Natl Acad Sci 78: 2869-2873

Pavlov MY, Watts RE, Tan Z, Cornish VW, Ehrenberg M \& Forster AC (2009) Slow peptide bond formation by proline and other $\mathrm{N}$-alkylamino acids in translation. Proc Natl Acad Sci 106: 50-54

Pelechano V \& Alepuz P (2017) elF5A facilitates translation termination globally and promotes the elongation of many non polyproline-specific tripeptide sequences. Nucleic Acids Res 45: 7326-7338

Pringle ES, McCormick C \& Cheng Z (2019) Polysome Profiling Analysis of mRNA and Associated Proteins Engaged in Translation. Curr Protoc Mol Biol 125: e79

Ripley BD (1988) Statistical Inference for Spatial Processes 1st ed. Cambridge University Press

Ross CA \& Tabrizi SJ (2011) Huntington's disease: from molecular pathogenesis to clinical treatment. Lancet Neurol 10: 83-98

Rossi D, Barbosa NM, Galvão FC, Boldrin PEG, Hershey JWB, Zanelli CF, Fraser CS \& Valentini SR (2016) Evidence for a Negative Cooperativity between elF5A and eEF2 on Binding to the Ribosome. PLOS ONE 11: e0154205

Saini P, Eyler DE, Green R \& Dever TE (2009) Hypusine-containing protein elF5A promotes translation elongation. Nature 459: 118-121

Saudou F \& Humbert S (2016) The Biology of Huntingtin. Neuron 89: 910-926

Schaffer M, Pfeffer S, Mahamid J, Kleindiek S, Laugks T, Albert S, Engel BD, Rummel A, Smith AJ, Baumeister W, et al (2019) A cryo-FIB lift-out technique enables molecular-resolution cryo-ET within native Caenorhabditis elegans tissue. Nat Methods 16: 757-762

Schmidt C, Becker T, Heuer A, Braunger K, Shanmuganathan V, Pech M, Berninghausen O, Wilson DN \& Beckmann R (2016) Structure of the hypusinylated eukaryotic translation factor elF-5A bound to the ribosome. Nucleic Acids Res 44: 1944-1951

Schuller AP \& Green R (2018) Roadblocks and resolutions in eukaryotic translation. Nat Rev Mol Cell Biol 19: 526-541 
Schuller AP, Wu CC-C, Dever TE, Buskirk AR \& Green R (2017) elF5A Functions Globally in Translation Elongation and Termination. Mol Cell 66: 194-205.e5

Tabrizi SJ, Ghosh R \& Leavitt BR (2019) Huntingtin Lowering Strategies for Disease Modification in Huntington's Disease. Neuron 101: 801-819

Tartari M, Gissi C, Lo Sardo V, Zuccato C, Picardi E, Pesole G \& Cattaneo E (2008) Phylogenetic comparison of huntingtin homologues reveals the appearance of a primitive polyQ in sea urchin. Mol Biol Evol 25: 330-338

Tauber E, Miller-Fleming L, Mason RP, Kwan W, Clapp J, Butler NJ, Outeiro TF, Muchowski PJ \& Giorgini F (2011) Functional Gene Expression Profiling in Yeast Implicates Translational Dysfunction in Mutant Huntingtin Toxicity. J Biol Chem 286: 410-419

The Huntington's Disease Collaborative Research Group (1993) A novel gene containing a trinucleotide repeat that is expanded and unstable on Huntington's disease chromosomes. Cell 72: 971-983

Ude S, Lassak J, Starosta AL, Kraxenberger T, Wilson DN \& Jung K (2013) Translation Elongation Factor EF-P Alleviates Ribosome Stalling at Polyproline Stretches. Science 339: 82-85

Vonsattel J-P, Myers RH, Stevens TJ, Ferrante RJ, Bird ED \& Richardson EP (1985) Neuropathological Classification of Huntington's Disease: $J$ Neuropathol Exp Neurol 44: 559-577

Wohlgemuth I, Brenner S, Beringer M \& Rodnina MV (2008) Modulation of the Rate of Peptidyl Transfer on the Ribosome by the Nature of Substrates. J Biol Chem 283: 32229-32235

Wu G-Q, Xu Y-M \& Lau ATY (2020) Recent insights into eukaryotic translation initiation factors $5 \mathrm{~A} 1$ and $5 \mathrm{~A} 2$ and their roles in human health and disease. Cancer Cell Int 20: 142

Yang J, Hao X, Cao X, Liu B \& Nyström T (2016) Spatial sequestration and detoxification of Huntingtin by the ribosome quality control complex. eLife 5: e11792 


\section{Acknowledgements}

We thank Pablo Sola Alarcón for valuable technical assistance. We would also like to thank Eber Martínez and the rest of the Animal facility at the Centro Nacional de Biotecnología (CNB-CSIC, Madrid) for their work over the years. qPCR experiments were provided by the Genomics and NGS Core Facility at the Centro de Biología Molecular Severo Ochoa which is part of the CEI UAM+CSIC, Madrid. The HPF and FS experiments together with the observation of brain sections were performed in the Electron microscopy facility at the CNB-CSIC. The acquisition of tilt series for tomography was performed in the Cryoelectron microscopy facility also at CNB-CSIC. We are profoundly grateful to the Fundación Ramón Areces for the grant that made this work possible and also AEI/FEDER (SAF2017-84565-R) for additional funds.

\section{Author Contributions}

EMS: Investigation, Validation, Formal Analysis, Methodology, Writing - Original draft .

IDL: Investigation, Resources, Writing - Review \& Editing.

IV: Investigation, Resources, Writing - Review \& Editing.

JJF: Conceptualization, Methodology, Software, Investigation, Formal Analysis, Writing - Original draft, Funding Acquisition.

MRFF: Conceptualization, Methodology, Investigation, Validation, Formal Analysis, Writing - Original draft, Funding Acquisition.

\section{Conflict of interest}

The authors declare that they have no conflict of interest in relation to this manuscript. 

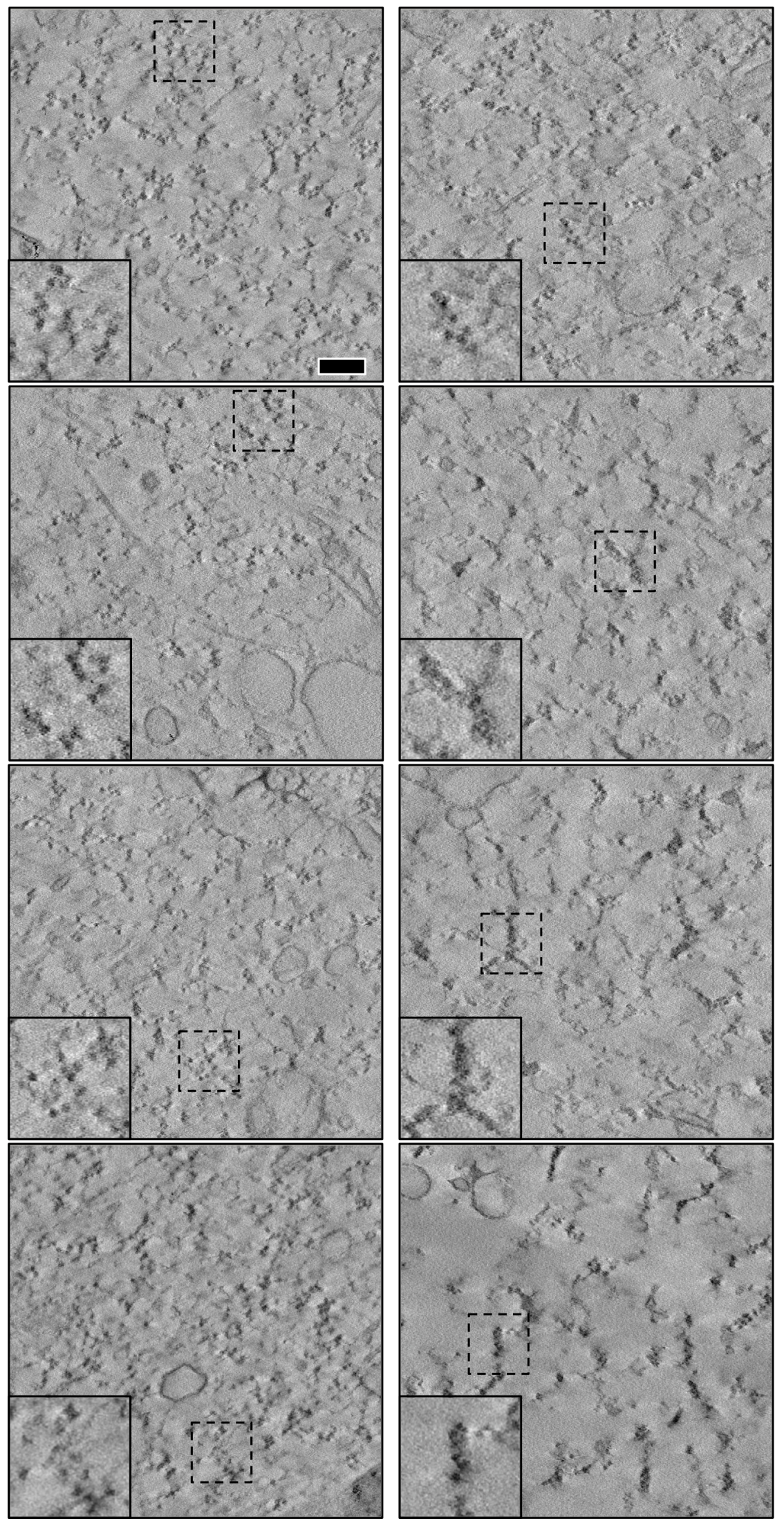

Figure 1. Progressive alterations in ribosomal/polysomal architecture in HD observed with ET. Slices of tomograms from WT animals (left) and HD animal model (right). From top to bottom: ages of 2, 8, 10, 11 months. Insets are magnified views of dashed boxes. Bar: $200 \mathrm{~nm}$. 


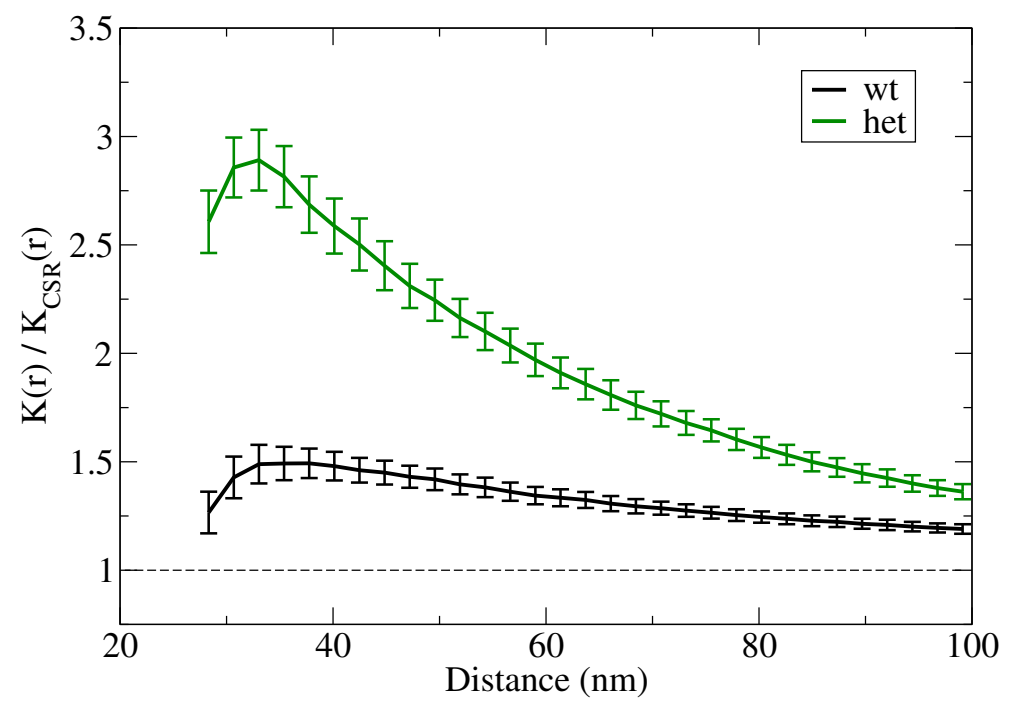

Figure 2. Second-order spatial pattern analysis based on Ripley's K function. Ripley's K-function relative to a random distribution, $K(r) / K_{C S R}(r)$ as a function of the distance calculated for tomograms from different neurons in the heterozygous HD model $(n=6)$ and wild-type animals $(n=5)$ at the age of 11-months. The plot shows average values and standard error. This plot represents the expected number of ribosomes within a distance $r$ around a random ribosome $(K(r))$, relative to the expected value in a random distribution $\left(K_{C S R}(r)\right)$. Values of the functions above 1 indicate clustering. 
A

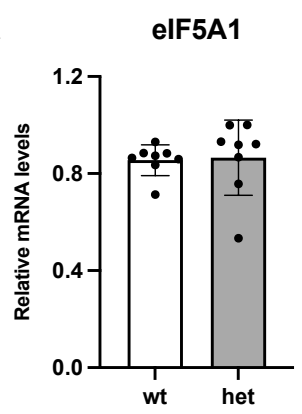

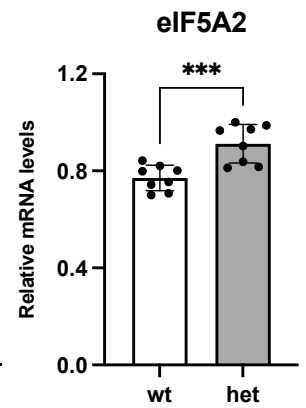
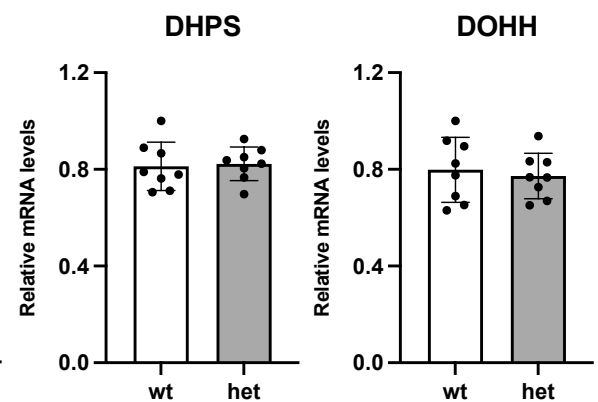

B
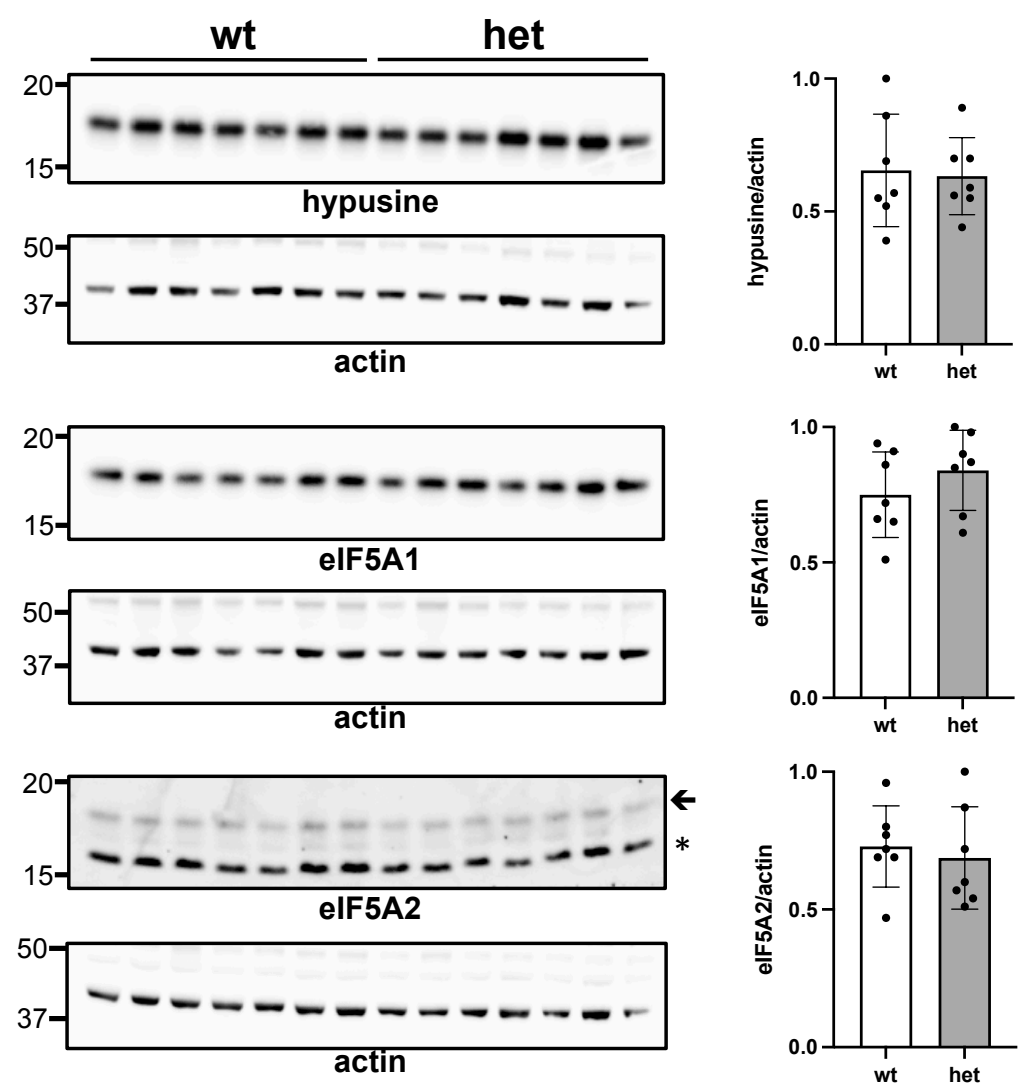

Figure 3. The elF5A2 stalling release factor is overexpressed in the striatum of heterozygous ZQ175 mice. (A) Relative accumulation of elF5A1, elF5A2, DHPS and $D O H H$ mRNA levels in the striatum of ZQ175 heterozygous and wt controls analyzed by qRT-PCR. The expression of the reference gene YWHAZ was used for normalization. For each independent gene the highest value after normalization with YWHAZ was set to 1 to facilitate comparisons. Data followed a normal distribution (Shapiro-Wilk test) with the exception of elF5A1. There is no significant difference in the expression of elF5A1, DHPS and DOHH genes $(p=0.2786,0.8130$ and 0.6642 respectively) but there is significant increase in the expression of the elF5A2 gene in the heterozygous animals $p=0.0009$. Bars show the mean $\pm S D, n=8(B)$ Western-blot analysis of the accumulation of hypusinated elF5A factor, elF5A1 and elF5A2 in the striatum of 12-month-old animals. The accumulation of actin was used as a loading control to quantify relative accumulation (plots on the right). All data sets follow a normal distribution. There is no significant difference in the accumulation of any of the factors ( $p=0.8289,0.2983$ and 0.6520 , respectively). Bars show the mean $\pm S D, n=7$. In elF5A2 arrow indicates the band at the expected size for full-length elF5A2. The asterisk indicates a very prominent band that could be a degradation product of elF5A2. 

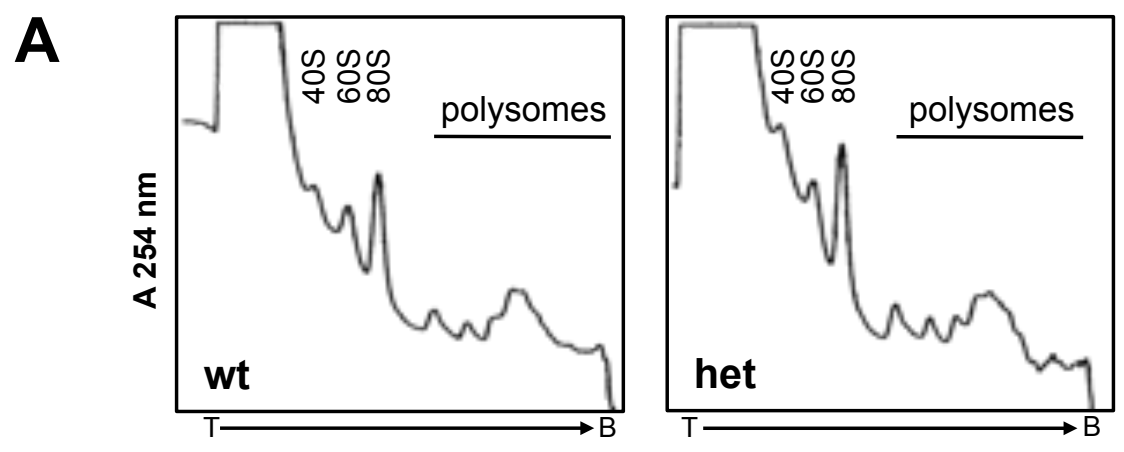

B
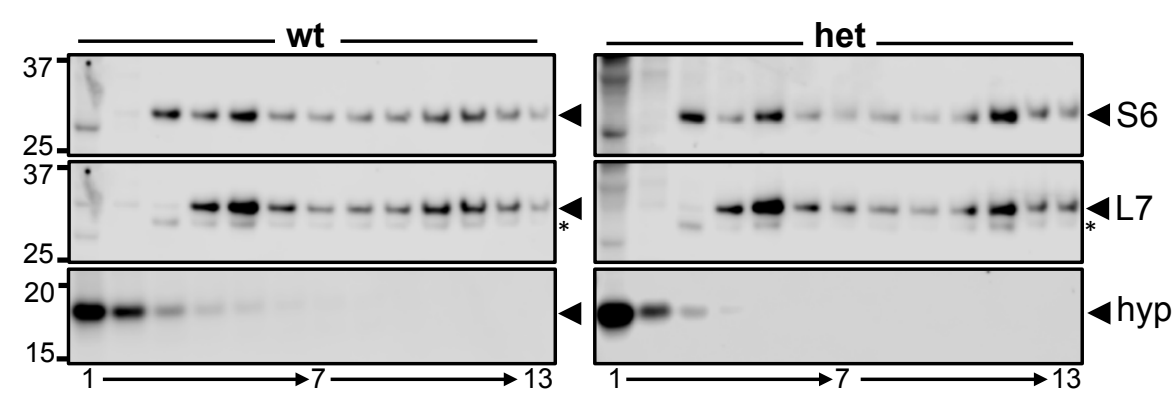

C
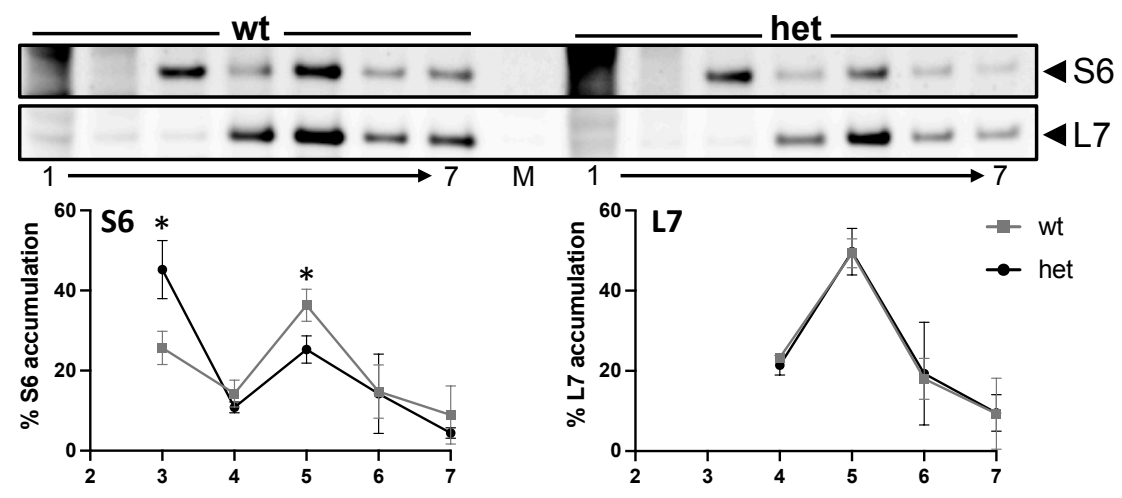

D
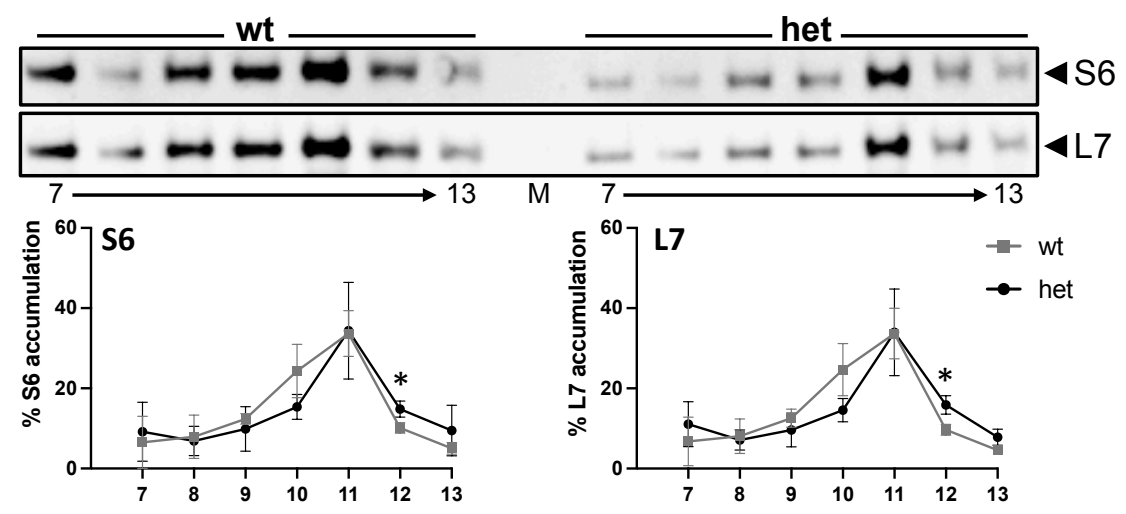

Figure 4. Polysomal sedimentation gradients of 9-month old wt and heterozygous zQ175 striatum. (A) Profiles obtained by continuous monitoring of absorbance at $254 \mathrm{~nm}$. 40S, $60 \mathrm{~S}$ and $80 \mathrm{~S}$ peaks and the polysomal are identifiable. In the $X$ axis, $T$ indicates the top and $B$ the bottom of the gradient (continuous $10-50 \%$ sucrose gradient). (B) Accumulation of ribosomal proteins RPS6 (S6) (small subunit) and RPL7 (L7) (large subunit) and hypusinated elF5A factor in gradient fractions by western-blot. Fraction 1 corresponds to the top and fraction 13 to the bottom of the gradient. The proportion of free 40S (represented by the accumulation of RPS6 in fraction 3) appears higher in the heterozygous gradients. The distribution of ribosomal 
proteins in the polysomal fractions is slightly different between both genotypes. Most of the hypusinated factor elF5A (>80\%) accumulates in the first two fractions that do not contain ribosomal subunits (soluble fractions). (C) To compare the relative accumulation of ribosomal proteins in wt and heterozygous striatal gradients, fractions 1 to 7 of wt and heterozygous gradients were loaded in the same gel. To compensate the absence of an internal quantity control, three independent gels were loaded with the same fractions. The percentage of accumulation was calculated for each protein and each genotype independently. $100 \%$ was set as the sum of the intensities of a given protein in a given genotype. Fractions 1 and 2 (for RPS6) and fractions 1, 2 and 3 (for RPL7) were not quantified as they do not accumulate a significant amount of the corresponding protein and they have high background signals. The graphics show the average percentage of accumulation $\pm S D$ for each fraction. The percentage of accumulation of the RPL7 protein (right panel) is highly overlapping in the wt and heterozygous fractions. However, the percentage of accumulation of the RPS6 protein (left panel) differs substantially. The quantification confirms that there is a significant increased in the percentage of RPS 6 protein in the heterozygous fraction 3 that coincides with free 40S) $(p=0.0156)$ while correspondingly there is a decreased percentage of $S 6$ in fraction $5(p=0.0223$ ) (attributed to $80 S$ subunits). (D) The relative accumulation of ribosomal proteins in fractions 7 to 13 was compared by loading the corresponding fractions and by quantification as explained in (C). The accumulation is very similar for RPS6 and RPL7 within each genotype. Most remarkable differences between wt and heterozygous patterns are an increased percentage of RPS6 and RPL7 accumulation in wt fraction 10 that does not reach statistical significance $(p=0.1038$ and $p=0.0708$, respectively). There is an increased percentage of accumulation of RPS6 and RPL7 in heterozygous fraction 12 that reaches statistical significance for both proteins ( $p=0.0265$ and $p=0.0164$, respectively). For (C) and (D) $M$ indicates the channel were the molecular weight markers were loaded. 

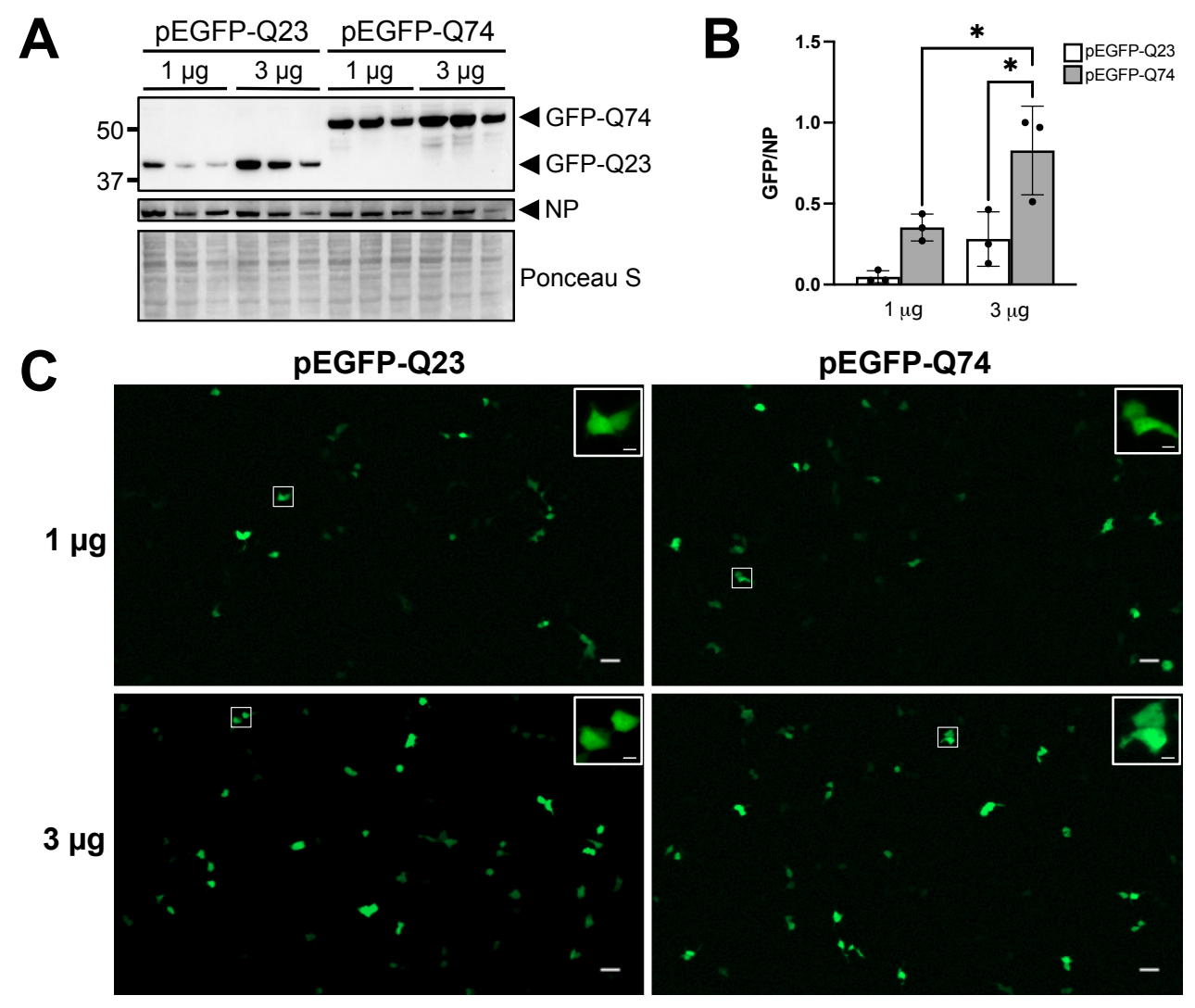

D
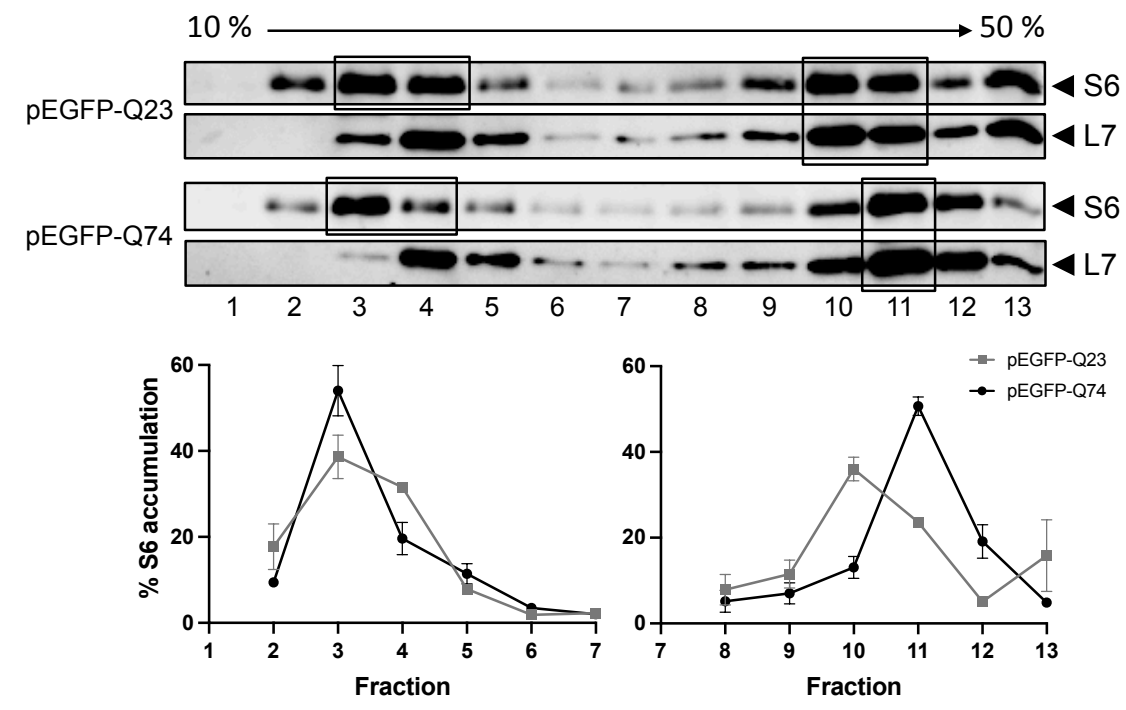

Figure 5. Transfection of HEK293 cells with plasmids expressing a GFP fusion protein containing part of the exon1 $H T T$ coding sequence including $23 Q$ or $74 Q$. (A) Western-blot analysis of the accumulation of fusion proteins GFP-Q23 and GFPQ74 at 18 hours post-transfection with different amounts of plasmids pEGFP-Q23 and pEGFP-Q74 (1 or $3 \mu \mathrm{g}$ per well). A band of the expected size was detected for each fusion protein. A plasmid expressing the NP protein was co-transfected for quantification purposes. The accumulation of the NP protein is shown in the second panel. The third panel shows the membrane staining with Ponceau S. Samples were 
run in triplicate. (B) Quantification of the relative accumulation of GFP-Q23 and GFPQ74 at the different transfection conditions shown in A. Mean values +/- SD are shown. There is an increase in the accumulation of GFP-Q74 at both plasmid concentrations, the difference is not significant at $1 \mu \mathrm{g}(\mathrm{p}=0.1000)$ but it is at $3 \mu \mathrm{g}(\mathrm{p}=0.0422)$. For both plasmids the increase in the amount of the transfected plasmid increases the accumulation of the fusion protein, this increase is not statistical significant for GFPQ23 $(p=0.1000)$ but it is for GFP-Q74 ( $p=0.0453)$. The distribution of the pEGFP-Q23 1 $\mu \mathrm{g}$ data set is not normal and for that reason all comparisons including this data set were compared using the non-parametric Mann-Whitney test. All the other data groups had a normal distribution and were compared by using the parametric unpaired t-test. (C) Fluorescence images to detect the accumulation of GFP fusion proteins by GFP fluorescence were taken on culture plates by using a Leica DMI6000B microscope at $10 x$ magnification. The insets show a zoom of the image to show that at this time posttransfection cells accumulate only soluble GFP fusion proteins. Percentages of transfection were: pEGFP-Q23 $1 \mu \mathrm{g} 3.4 \%$, pEGFP-Q74 $1 \mu \mathrm{g} 3.5 \%$, pEGFP-Q23 $3 \mu \mathrm{g}$ $5.4 \%$ and pEGFP-Q74 $3 \mu \mathrm{g} 5 \%$. Scale bar is $50 \mu \mathrm{m}$ for general panels and $10 \mu \mathrm{m}$ for insets. (D) Western-blot analysis of fractions from polysomal sedimentation gradients obtained from HEK293 cells transfected either with pEFGP-Q23 or pEGFP-Q74. Cells were recovered 24 hours post-transfection and the percentage of transfection was 20$25 \%$. Boxes on the left highlight the accumulation of RPS6 (small ribosomal subunit) in the first fractions of the gradient. The difference in the distribution of RPS6 remarks that the overexpression of the fusion protein containing $74 \mathrm{Q}$ induces an increased percentage of free $40 \mathrm{~S}$ subunits as observed in gradients of striatal samples derived from the zQ175 mouse model. Boxes on the right side of the western-blots highlight a different percentage of accumulation of polysomes in the mid polysomal area that is also similar to the obtained gradients from striatal samples, with pEGFP-Q74 having a peak of accumulation around fraction 11. The bottom graphs show the percentage of accumulation of RPS6 protein in the different fractions to highlight differences. Two sets of equivalent western-blots were run to compensate the absence of a shared internal loading control. The graphs show the percentage mean \pm SEM. 


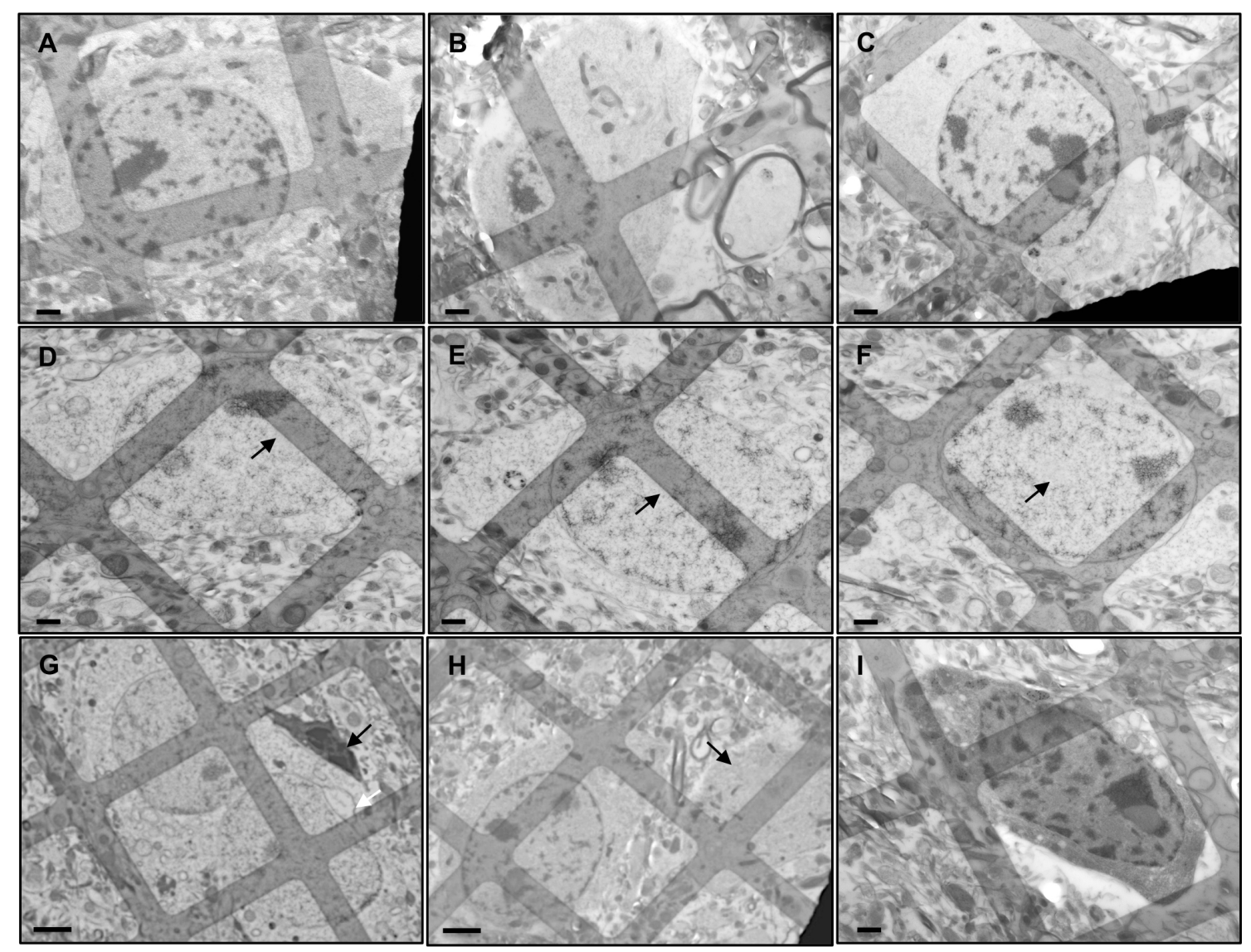

Figure EV1. Representative EM images of striatal cells from $250 \mathrm{~nm}$ thick sections of brain tissue. (A-F) Cells compatible with medium-sized spiny neurons (MSSN) based on morphological criteria in wt control (A-C) and heterozygous zQ175 (D-F) animals at an age of 11 months. In the HD model, these cells in general look paler, as seen in the chromatin, nucleus and cytoplasm. In addition, nuclear inclusions are identifiable in the HD model (arrows in D-F). (G) A cell, likely microglia, with nucleus and cytoplasm that are highly dense (black arrow) is observed near two cells compatible with MSSN that exhibit pale appearance. One of these MSSN has a nuclear bleb (white arrow), a mark often found in HD model neurons and indicative of neurodegeneration. (H) A putative MSSN and, on the right, a portion of cytoplasm (arrow) with similar appearance but without the presence of nucleus. (I) A cell from a control animal, with size and morphology compatible with a MSSN, shows highly dense nucleus and cytoplasm. Bars: $1 \mu \mathrm{m}(\mathrm{A}-\mathrm{F}, \mathrm{I}), 2 \mu \mathrm{m}(\mathrm{G}-\mathrm{H})$. 


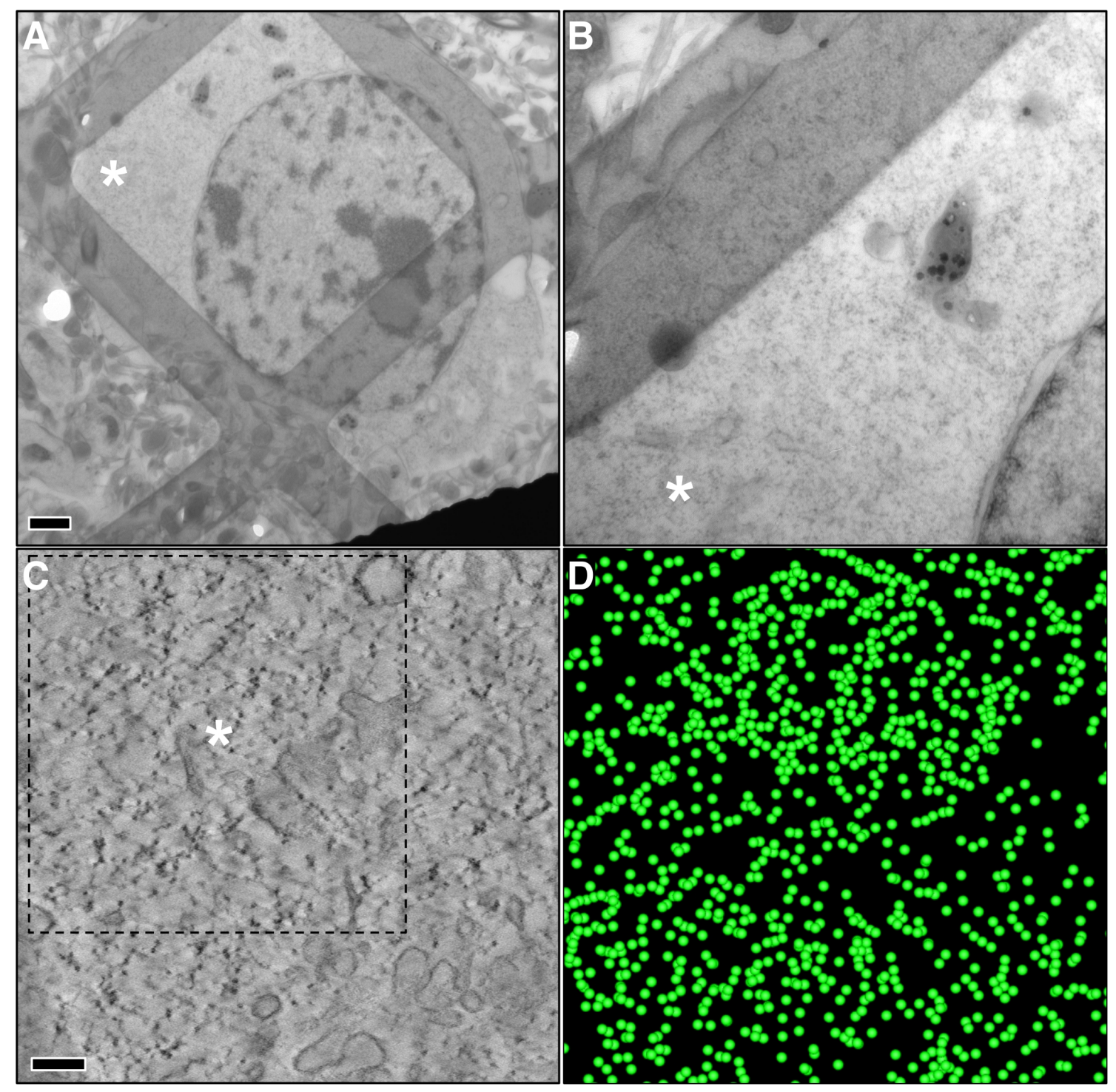

Figure EV2. Electron tomography of a striatal neuron from a $250 \mathrm{~nm}$ thick section of brain tissue of an 11 month-old wild-type mouse. (A) Image from conventional 2D electron microscopy, showing the nucleus of the neuron and cytoplasm. Bar: $1 \mu \mathrm{m}$. (B) Magnified view from the area marked by the asterisk in (A). (C) Slice of the tomogram that was computed from a tilt-series acquired at the position marked by the asterisk in (B). The individual ribosomes are clearly identifiable as black dots in the spotty cytoplasm. The cytoplasm is crowded by numerous ribosomes, and polysomal clusters are also observed. Bar: $200 \mathrm{~nm}$. (D) 3D visualization of the ribosomes detected by our automated procedure based on Laplacian of Gaussian (LoG) and represented by green spheres. These ribosomes correspond to the area dashed in (C). 


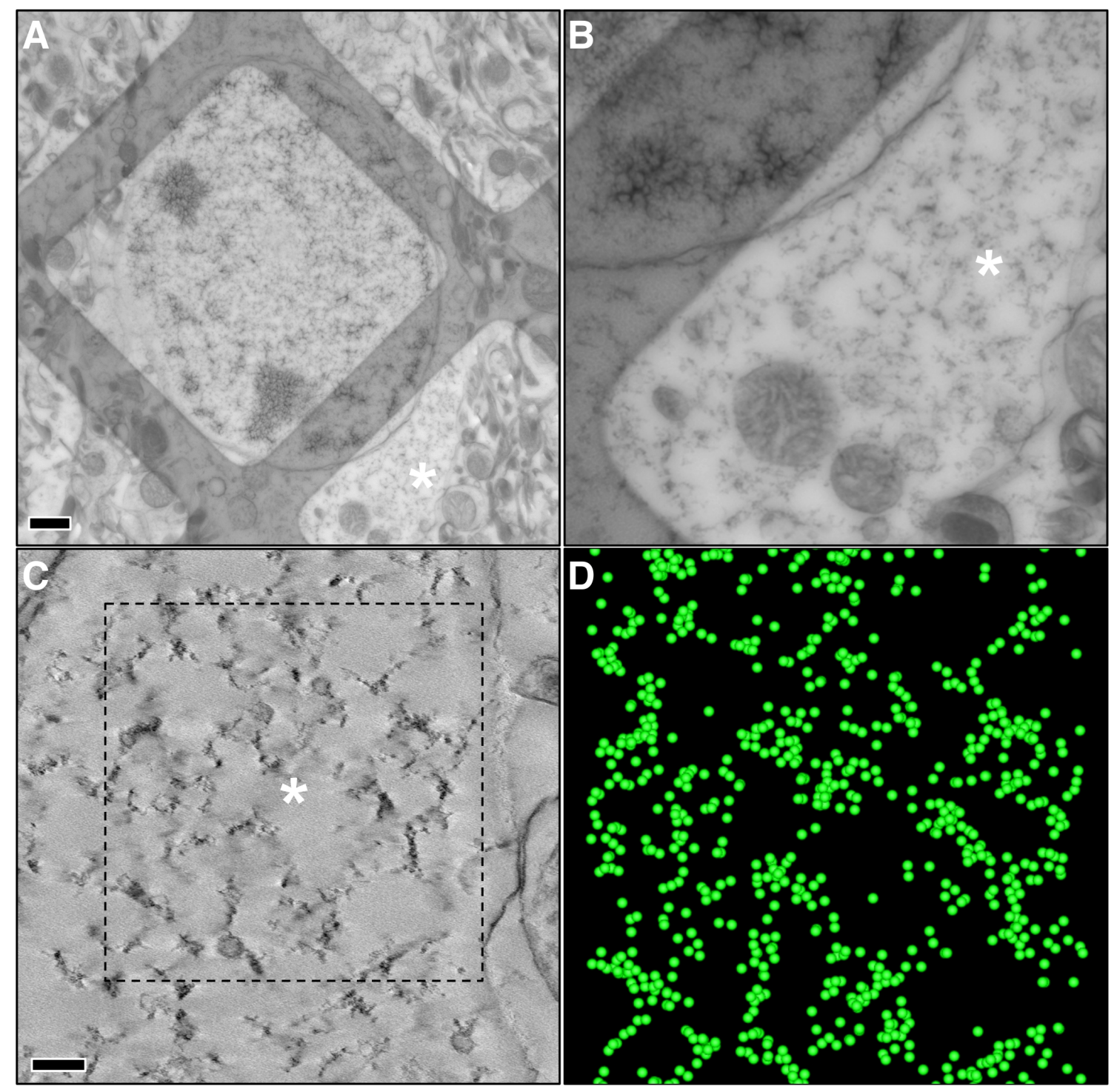

Figure EV3. Electron tomography of a striatal neuron from a $250 \mathrm{~nm}$ thick section of brain tissue of an 11 month-old zQ175 heterozygous HD animal model. (A-D) Interpretation of the panels is as described in Figure EV2. In this case, individual ribosomes are hardly observed in the tomogram (C). Instead, tightly-coupled clusters of ribosomes are observed, and there is substantial empty cytoplasmic space among the clusters. 


\section{Supplementary Figures}

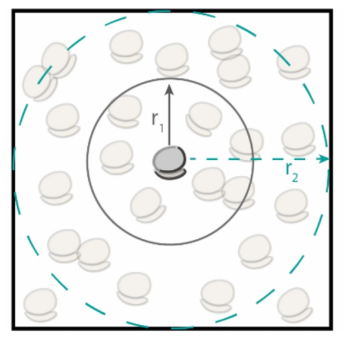

Ripley's K function

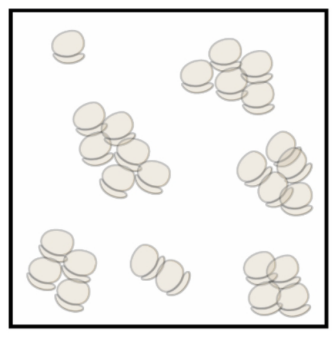

$\mathrm{K}(\mathrm{r}) / \mathrm{K}_{\mathrm{CSR}}(\mathrm{r})>1$

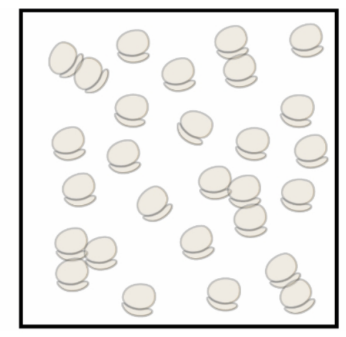

$\mathrm{K}(\mathrm{r}) / \mathrm{K}_{\mathrm{CSR}}(\mathrm{r})=1$

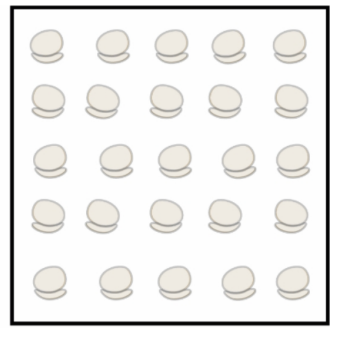

$\mathrm{K}(\mathrm{r}) / \mathrm{K}_{\mathrm{CSR}}(\mathrm{r})<1$

Figure S1. Ripley's K function and spatial distribution patterns. Left: Ripley's K function is calculated at a range of distances $r$ and measures the expected number of ribosomes within the distance $r$ from an arbitrary ribosome, normalized by the density of the ribosome distribution. In this work, $\mathrm{K}$ function has been implemented in 3D, though for simplicity the sketch is 2D. Right: Three main spatial distribution patterns can be identified when comparing the actual $\mathrm{K}$ function with the expected value under complete spatial randomness $\left(\mathrm{K}_{\mathrm{CSR}}\right)$, namely, clustering $\left(\mathrm{K}(\mathrm{r}) / \mathrm{K}_{\mathrm{CSR}}(\mathrm{r})>1\right)$, randomness $\left(\mathrm{K}(\mathrm{r}) / \mathrm{K}_{\mathrm{CSR}}(\mathrm{r})=1\right)$ or regularity or dispersion $\left(\mathrm{K}(\mathrm{r}) / \mathrm{K}_{\mathrm{CSR}}(\mathrm{r})<1\right)$. 


\section{Supplementary Tables}

Table 1. Primers employed for qRT-PCR of the target genes of interest

\begin{tabular}{|c|c|c|c|c|}
\hline Gene & ENSEMBL & Primer & Sequence 5 '- 3' & Amplicon size \\
\hline \multirow{2}{*}{ elF5A1 } & \multirow{2}{*}{ ENSMUSG00000078812 } & $\mathrm{Fw}^{1}$ & GGATGTCCCCAACATCAAAC & \multirow{2}{*}{$75 \mathrm{bp}$} \\
\hline & & $\mathrm{Rv}^{2}$ & TCCTGGAGCAGGGATAGGTA & \\
\hline \multirow{2}{*}{ elF5A2 } & \multirow{2}{*}{ ENSMUSG00000050192 } & Fw & GCAAAATCGTGGAGATGTCA & \multirow{2}{*}{127 bp } \\
\hline & & $\mathrm{Rv}$ & TCCATGTTGTGAGTAGAAGGACA & \\
\hline \multirow{2}{*}{ DHPS } & \multirow{2}{*}{ ENSMUSG00000060038 } & Fw & TACCTCGTGCAGCACAACA & \multirow{2}{*}{102 bp } \\
\hline & & Rv & GAACTCGCCAAGGTATGTGG & \\
\hline \multirow{2}{*}{$\mathrm{DOHH}$} & \multirow{2}{*}{ ENSMUSG00000078440 } & Fw & CCGGGCCCTGTTTACACT & \multirow{2}{*}{$60 \mathrm{bp}$} \\
\hline & & Rv & CGGCTGATCCACGAGATAG & \\
\hline
\end{tabular}

${ }^{1} \mathrm{Fw}$ : forward primer

${ }^{2} \mathrm{Rv}$ : reverse primer

Table 2. Primers employed for the amplification by qRT-PCR of reference genes for normalization

\begin{tabular}{|l|l|l|l|}
\hline \multirow{2}{*}{ Gene } & Primer & Sequence 5 '- 3' & \multirow{2}{*}{ Amplicon size } \\
\hline \multirow{2}{*}{$18 S$} & $\mathrm{Fw}^{1}$ & CTCAACACGGGAAACCTCAC & \multirow{2}{*}{$110 \mathrm{bp}$} \\
\cline { 2 - 4 } & $\mathrm{Rv}^{2}$ & CGCTCCACCAACTAAGAACG & \\
\hline \multirow{2}{*}{ BACT } & $\mathrm{Fw}$ & CTAAGGCCAACCGTGAAAAG & \multirow{2}{*}{$104 \mathrm{bp}$} \\
\cline { 2 - 4 } & $\mathrm{Rv}$ & ACCAGAGGCATACAGGGACA & \\
\hline \multirow{2}{*}{ B2M } & $\mathrm{Fw}$ & TACATACGCCTGCAGAGTTAAGCA & \multirow{2}{*}{$76 \mathrm{bp}$} \\
\cline { 2 - 4 } & $\mathrm{Rv}$ & TGATCACATGTCTCGATCCCAG & \\
\hline \multirow{2}{*}{ GAPDH } & $\mathrm{Fw}$ & CACCACCAACTGCTTAGCCC & \multirow{2}{*}{$76 \mathrm{bp}$} \\
\cline { 2 - 4 } & $\mathrm{Rv}$ & TGTGGTCATGAGCCCTTCC & \\
\hline \multirow{2}{*}{ YWHAZ } & $\mathrm{Fw}$ & TTACTTGGCCGAGGTTGCT & \multirow{2}{*}{$60 \mathrm{bp}$} \\
\cline { 2 - 4 } & $\mathrm{Rv}$ & TGCTGTGACTGGTCCACAAT & \\
\hline
\end{tabular}

${ }^{1}$ Fw: forward primer

${ }^{2}$ Rv: reverse primer 